\title{
The Philosophy of
}

\section{Perception}

An explanation of Realism, Idealism and the Nature of Reality 


\section{Best Publications Limited}

14 Longcroft Terrace

Newlands

Wellington

New Zealand

\section{Copyright (C) 2017 Rochelle Forrester}

All Rights Reserved

The moral right of the author has been asserted

Anyone may reproduce the whole or any part of this paper without the permission of the author, so long as a full acknowledgement of the source of the reproduced material is made

Short Edition of Sense Perception and Reality

Published 15 March 2017 
This is the short edition of Sense Perception and Reality, the first edition being published on my website at www.homepages.paradise.net.nz/rochelle.f. The second edition, including new material on animal senses, neurology and cognitive psychology and the debate between Bohr and Einstein on quantum theory and alternatives to the Copenhagen Interpretation of quantum theory, was published as a hard copy book and also on websites such as academia.edu, Figshare, Mendeley, Vixra and the Social Science Research Network. Sense Perception and Reality has also been published as an e-book on Kindle and other formats. The short edition contains much reduced material on quantum mechanics and non-Euclidean geometry and is intended to be more readable and more user friendly than the earlier editions. Related material on quantum mechanics is contained in my paper The Quantum Measurement Problem: Collapse of the Wave Function explained available on academia.edu, Figshare, $\underline{\text { Vixra, Humanities Commons and the Social Science Research Network. }}$

I had three main aims when writing this paper. The first is to change the way we think of the macro world. The second is to bring the macro world and the quantum world together and show how they both operate under the same basic rules. The third is to suggest a vision of the universe based upon modern research on animal senses, neurology and cognitive psychology and the Copenhagen Interpretation of quantum mechanics.

This paper is a paper on metaphysics in that it sits next to the General Theory of Relativity as a description of the universe. General Relativity gives an astrophysics description of the universe using the human sense of sight, while Sense Perception and Reality and The Philosophy of Perception describe the universe in a wider sense embracing sense perception in all conscious beings. Sense Perception and Reality and The Philosophy of Perception are scientific in that they use the facts of animal perception, neurology and cognitive psychology and provide rational arguments from these facts to produce a wider explanation of the universe than is provided by General Relativity. It sits on the borders of science and philosophy and incorporates material from both science and philosophy. 
Other papers written by Rochelle Forrester concern the study of social and cultural history and the placing of cultural evolution, social change and philosophy of history on a scientific basis capable of rational analysis and understanding. This has resulted in a hard copy book How Change Happens: A Theory of Philosophy of History, Social Change and Cultural Evolution and a website How Change Happens Rochelle Forrester's Social Change, Cultural Evolution and Philosophy of History website. There are also philosophy of history papers such as The Course of History, The

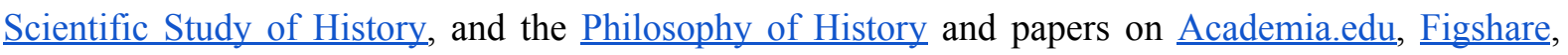
Humanities Commons, Mendeley, Open Science Framework, Orchid, Phil Papers, SocArXiv, Phil

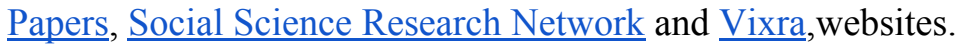

The study of social and cultural history and social change includes a study of the history of science and technology due to the affect scientific and technological discoveries have on social and cultural history. A number of papers have been written on scientific and technological discoveries and the effect they have had on social change and cultural evolution and those papers are below:

\begin{tabular}{|c|c|c|c|}
\hline \multicolumn{4}{|c|}{ The Invention of Stone Tools } \\
\hline The Invention of Glass & \multicolumn{3}{|c|}{$\begin{array}{l}\text { The Development of Agriculture and Pastoralism } \\
\text { History of Astronomy } \quad \text { Invention of Microscopes and Telescopes }\end{array}$} \\
\hline History of Printing & \multicolumn{2}{|c|}{ The Discovery of Steam Power } & History of Electricity \\
\hline Electric Telegraph & Telephone $\quad \underline{\text { Radio }}$ & $\underline{\text { Television }}$ & $\underline{\text { Motion Pictures }}$ \\
\hline \multicolumn{2}{|c|}{ 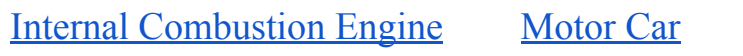 } & Aeroplanes The Hist & Medicine \\
\hline \multicolumn{4}{|c|}{ The Discovery of the Periodic Table $\quad$ The Discovery of the Atomic World } \\
\hline
\end{tabular}

Other papers by Rochelle Forrester include works on Epistemology and the Philosophy of Perception such as Sense Perception and Reality and on quantum mechanics such as the Quantum Measurement Problem and The Bohr and Einstein debate on the meaning of quantum physics. Rochelle Forrester's

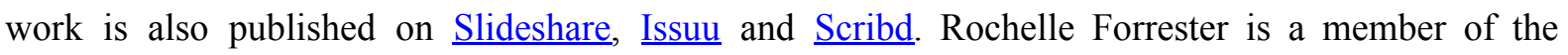
International Network for Theory of History. 


\section{Table of Contents}

$\begin{array}{ll}\text { Part I Perceptual Relativity } & 5\end{array}$

$\begin{array}{ll}\text { The problem } & 6\end{array}$

$\begin{array}{lr}\text { Animal senses } & 10\end{array}$

$\begin{array}{lr}\text { Neurology and cognitive psychology } & 18\end{array}$

$\begin{array}{ll}\text { Perceptual relativity } & 23\end{array}$

Arguments against the argument from perceptual relativity 28

$\begin{array}{ll}\text { Reid's solution } & 33\end{array}$

\begin{tabular}{ll} 
Quantum mechanics & 37 \\
\hline
\end{tabular}

Criticism of the observer dependent world 41

Alternatives to the Copenhagen Interpretation 42

Part II The consequences of perceptual relativity and the observer dependent universe 46

$\begin{array}{ll}\text { Bibliography } & 51\end{array}$

Philosophy and Perception $\quad 51$

Quantum theory 53 


\title{
The Philosophy of Perception:
}

\section{An explanation of Realism, Idealism and the Nature of Reality}

\author{
Short edition of Sense Perception and Reality \\ By Rochelle Forrester
}

\begin{abstract}
This paper investigates the nature of reality. It will do so by looking at the philosophical debate between realism and idealism and at scientific investigations in quantum physics. The concept of perceptual relativity will be examined and this will involve looking at sense perception in other animals, and at neurology and cognitive psychology, and at various examples of perceptual relativity in science. It will be concluded that the universe is observer dependent and that there is no reality independent of the observer, which is knowable to the observer. The paper concludes by an investigation of what an observer dependent universe would be like and that recognition of an observer dependent world would lead to a much more open minded and tolerant world.

The paper will describe the nature of reality as we can understand it through our sensory apparatus. It will argue that it is only through our sensory apparatus that we can know the real or external world. It will also argue that there are many and possibly an infinite number of realities each as valid and true as any other. Each reality is not something which is out there in the physical world, if such a world exists, but consists of a relationship between the sensory apparatus and the physical world. The world we know is observer dependent and changes in the sensory apparatus used to perceive the world will result in changes in the world we know. This situation exists both in our macro world and in the micro world of quantum physics. Animals with different sensory apparatus receive different sensory perceptions from each other and from those received by human beings. Each animal's sense perceptions can be regarded as a set and each set of sense perceptions constitute that animal's world. Every individual's world within a species will be very similar but not identical; but the worlds of different species may vary greatly. However each species world is as valid as any other species world, so that there is no single objective reality but rather a great variety of subjective realities each as valid as the other.
\end{abstract}

\section{Part 1}

\section{Perceptual relativity}

Philosophers have been studying the problem of how we can know the world for thousands of years. Our method of knowing about the world is primarily and perhaps exclusively through our 
sensory apparatus. The traditional senses of sight, hearing, touch, taste and smell provide us with information as to what there is in the world. Additional information is provided by non-traditional senses such as the senses of balance, hunger, temperature and pain. This information, whether from traditional or non-traditional senses, is usually called sensations, sense perceptions or sense data, lets us know the colour and shape of things, any sound they may make, how hard or soft things are and what they taste or smell like. They allow us to be aware of certain qualities of things in the world. There appears to be no way in which we can find out about the world external to ourselves, other than through our sensory apparatus. There is no knowledge known before or apart from sense perceptions. A priori knowledge does not exist; it is just a philosophical fantasy.

In this paper I shall refer to sensations, sense perceptions or sense data, by which I mean the colors, shapes, sizes, smells, sounds, tastes and feelings which our senses provide us with. Sensations are the raw material of perception; it is that which is perceived with no inference or judgment being made upon it. It is something about which we cannot be mistaken; it is simply that which is given by our sensory apparatus, without it being processed in any way other than that required for awareness of the sensations. It would be unwise to deny the existence of sensations as that would involve denying we see colors, shapes, sizes, hear sounds and perceive smells, tastes and feel things. Further definitions are "sensory apparatus" means sense organs, and the brain and everything else that is involved in the process of giving us awareness of the external world. By "realism" I mean a view of an external world that is the same for all observers and is independent of observers, whether knowable through direct perception, or deductible from direct perception by means of science or mathematics or any other means.

\section{The Problem}

However, as has been noted in the past, sensations can be inconsistent and contradictory. This is sometimes called the problem of perceptual relativity. A fine example of this is provided by Bertrand Russell in The Problems of Philosophy when, referring to a table he states:

"To the eye it is oblong, brown, and shiny, to the touch it is smooth and cool and hard; when I tap it, it gives out a wooden sound. Anyone else who sees and feels and hears the table will agree with this description..."

But

"Although I believe that the table is really of the same colour all over, the parts that reflect the light look much brighter than the other parts, and some parts look white because of reflected light. I know that, if I move, the parts that reflect the light will be different, so that the apparent distribution of colours on the table will change. It follows that if several people are looking at the table at the same moment, no two of them will see exactly the same distribution of colours, because no two can see it from exactly the same point of view, and any change in the point of view makes some change in the way the light is reflected...."

Russell goes on to say: 
"It is evident from what we have found, that there is no colour which pre-eminently appears to be the colour of the table, or even of any one particular part of the table-it appears to be of different colours from different points of view, and there is no reason for regarding some of these as more really its colour than others. And we know this even from a given point of view the colour will seem different by artificial light, or to a colour-blind man, or to a man wearing blue spectacles, while in the dark there will be no colour at all, though to touch and hearing the table will be unchanged. This colour is not something which is inherent in the table, but something depending upon the table and the spectator and the way the light falls on the table. When, in ordinary life, we speak of the colour of the table, we only mean the sort of colour which it will seem to have to a normal spectator from an ordinary point of view under usual conditions of light. But the other colours which appear under other conditions have just as good a right to be considered real; and therefore, to avoid favouritism, we are compelled to deny that, in itself, the table has any one particular colour.

The same thing applies to the texture. With the naked eye one can see the grain, but otherwise the table looks smooth and even. If we looked at it through a microscope, we should see roughness and hills and valleys, and all sorts of differences that are imperceptible to the naked eye. Which of these is the "real" table? We are naturally tempted to say that what we see through the microscope is more real, but that in turn would be changed by a still more powerful microscope. If, then, we cannot trust what we see with the naked eye, why should we trust what we see through a microscope? Thus, again, the confidence in our senses with which we began deserts us.

The shape of the table is no better. We are all in the habit of judging as to the 'real' shapes of things, and we do this so unreflectingly that we come to think we actually see the real shapes. But, in fact, as we all have to learn if we try to draw, a given thing looks different in shape from every different point of view. If our table is 'really' rectangular, it will look, from almost all points of view, as if it had two acute angles and two obtuse angles. If opposite sides are parallel, they will look as if they converged to a point away from the spectator; if they are of equal length, they will look as if the nearer side were longer. All these things are not commonly noticed in looking at a table, because experience has taught us to construct the 'real' shape from the apparent shape, and the 'real' shape is what interests us as practical men. But the 'real' shape is not what we see; it is something inferred from what we see. And what we see is constantly changing in shape as we move about the room; so that here again the senses seem not to give us the truth about the table itself, but only about the appearance of the table.

Similar difficulties arise when we consider the sense of touch. It is true that the table always gives us a sensation of hardness, and we feel that it resists pressure. But the sensation we obtain depends upon how hard we press the table and also upon what part of the body we press with; thus the various sensations due to various pressures or various parts of the body cannot be supposed to reveal directly any definite property of the table, but at most to be signs of some property which perhaps causes all the sensations, but is not actually apparent in any of them. And the same applies still more obviously to the sounds which can be elicited by rapping the table.

Thus it becomes evident that the real table, if there is one, is not the same as what we immediately experience by sight or touch or hearing. The real table, if there is one, is not immediately known to us at all, but must be an inference from what is immediately known."

(Russell, 1912, 2-4)

The example given by Russell relates only to the human sensory apparatus, yet it is apparent many other animals perceive things differently from the way humans perceive them. Some animals 
can see colors that humans cannot see, while others can see only in black and white. Dogs can sense smells and hear sounds that are not perceivable by humans; other animals appear to be able to detect the earth's magnetic field; while some fish generate electric fields and are able to detect disturbances in their fields. What is perceived seems to vary with the sensory apparatus used for the perception.

One reason for considering animal senses is that it raises certain questions of what may be considered to be standard observers. Some philosophers consider that a standard observer in relation to colors would be a human with no color blindness. This observer will obtain the correct view of colors; he or she would see reality as it is. However some animals are naturally color blind and others see a different range of colors from humans, so their view of colors will differ radically from that of the standard human observer. It would be difficult to justify why the human view should be preferred over that of other animals and be considered the real or correct view of the world.

Astronomy provides us with other examples of the unreliability of our senses. Our naked senses tell us the Earth is motionless and each day the Sun travels around the Earth. This was the view of the universe held by humans for most of humanity's existence. Johannes Kepler suggested the Earth spun on its axis and orbited the Sun in an elliptical orbit. This view was later confirmed by observations made by the newly invented telescope. Einstein, using still more accurate observations, concluded the Earth orbited the Sun in circular orbits in curved, four dimensional, space-time. We also believe the Earth is traveling through space as part of an expanding universe. Each view of the Earth's motion was based on the best observations possible at the time. Different instruments, naked senses, telescopes and superior telescopes produced different observations which produced different views as to the Earth's motion. It may be one day we will be able to establish a completely certain view of the Earth's movement, but considering the way in which previous theories were completely accepted and then later discarded, it would seem unwise to regard any theory as the correct and final view.

The subatomic world provides us with another scientific example of how misleading our sensory apparatus can be. Objects often appear solid to us but science tells us they are largely made up of empty space containing tiny particles called electrons, protons and neutrons. Which is correct the scientific view or the view provided by our unaided sensory apparatus? Modern humans will usually prefer the scientific view, but for nearly all of human history it was accepted that objects that appeared solid, were solid. There is no reason in principle that there could not be a being who could directly see subatomic particles and the space between them and not see the objects we see. The scientific view is simply obtained by using the normal human sensory apparatus and adding certain scientific instruments and experiments and a logical analysis of the results of those experiments. Different sensory apparatus will give different views of the universe and adding scientific instruments and experiments to a sensory apparatus, amounts to changing the sensory apparatus.

The Doppler Effect provides a further example of differing observations by different observers. The Doppler Effect shows up both in our senses of hearing and vision. We experience it in everyday life as the increase in the pitch of a police siren as it approaches us and the fall in the pitch after it has passed and is moving away from us. This is because as the police car approaches the sound waves are bunched up or shortened and as the car moves away the sound waves are stretched out. Our ears interpret different lengths of sound waves as different pitches, the shorter the wave the higher the pitch. A similar effect applies if the source of the sound is stationary and the perceiver is moving. The Doppler Effect also applies to light and all other electro-magnetic radiation, but does not show up as a visible difference in colour, but can be seen as a shift in the spectral lines within electro-magnetic radiation. As the universe seems to be in continuous motion; so the Doppler Effect will always apply. It cannot be said that sound has any single, true or real pitch or radiation has any single, true or real 
spectral lines. It may be argued that there are single, real or true pitches or spectral lines at particular motions for observers of light or sound sources. This however does not give real spectral lines or real pitches. Spectral lines and pitches still vary with the observer.

A further illustration of the problem is shown by the phenomena of space contraction in the theory of special relativity. If an observer is moving away from a scene at the end of a street, at the speed of light, the scene will remain fixed and all activity in the street will cease. Such an observer will only see the scene available to the observer immediately before the observer began moving away at the speed of light. If the observer is moving at close to the speed of light the objects in the street will appear to change shape. They will tend to appear thinner and taller, buildings and people will seem crowded together as horizontal distances will appear shorter. The same effect is seen by the stationary observer when observing the person moving away at close to the speed of light. The person moving at the speed of light will appear to contract along the direction of motion, the person will appear thinner and taller. This effect has been observed in particle accelerators where protons which normally behave as spheres tend to flatten out as they get closer to the speed of light and assume the shape of a flattened ellipsoid. Space contraction is actually taken into account when constructing particle accelerators; without it particle accelerators would not work. One situation when space contraction does not occur is when the observer watching the object moving at close to the speed of light, is also moving at the same speed.

The fact that objects change shape when moving at close to the speed of light and objects in any stationary scene observed by a person moving at close to the speed of light change shape, again raises the question of what is the real shape of the objects involved. Their shape seems to alter with the movement of the observer and due to the movement of the objects themselves. In a universe where everything seems to be moving, the earth spins on its axis and orbits the sun and our galaxy flies through the universe as part of an expanding universe, it does not seem possible to give a privileged position to stationary objects and observers. Of course objects and observers in our world do not move at the speed of light but if you are trying to discover the nature of the universe it is the principle that matters. This is because although our instruments and sensory apparatus are unable to detect the effects of special relativity at slow speeds, those effects are still there. The problem is that our instruments and sensory apparatus are just not good enough to pick up relativistic effects at slow speeds.

A further example of perceptual relativity is time dilation. Time dilation is another consequence of special relativity and involves time passing at different speeds for observers traveling at different speeds. If an observer is traveling away from a scene at the speed of light the scene will remain motionless and time will have ceased for that observer. This is because light reflected from the scene will never reach an observer moving away from the scene at the speed of light. Yet for a stationary observer the scene will change and time will pass at a normal rate. An observer traveling at half the speed of light will observe time passing but at a different and slower rate than the stationary observer. The effect of this is that there is no universal time; time varies according to the motion of the observer. Identical clocks carried by the three observers, would all record different times. None of these times would be any more correct than the other times. The only thing that is the same for all observers, according to the theory of special relativity, is the speed of light. However no observer actually sees the speed of light.

Some people may suggest we can use the laws of nature or our scientific laws to tell us about reality, but this will not work because those laws are dependent upon our observations and our observations are unreliable and contradictory. Our observations are necessarily a priori to our scientific laws and any defects in our observations will be carried over into our scientific laws. The 
fact that the human sensory apparatus gives all humans a very similar (but not identical) view of the world, but a view similar enough for most humans to be able to agree on the scientific laws, does not mean that those laws are necessarily correct and will enable us to understand the real world. A completely different sensory apparatus would produce different observations and would result in quite different scientific laws than those produced from observations made with the human sensory apparatus. A wide variety of completely different sensory apparatus can be seen in the animal world.

The current generally accepted view of the universe based upon human senses is general relativity. It describes how humans see the universe as the sense of sight is the most important human sense. A different animal with different senses may produce an entirely different view of the universe. A super intelligent dog or any other animal with a very well developed sense of smell may well develop a theory of the universe based on the sense of smell. Such a theory could be every bit as good a description of the universe as general relativity but would be utterly beyond human understanding. Our understanding of the universe is completely dependent upon the sensory apparatus available to us. Different animals have different sensory apparatus and so will have different but equally valid views of the universe. It is only by going outside our own sensory apparatus, and studying how other sensory apparatus work, that we are able to get a better understanding of our own sensory apparatus. Studying other animal's sensory apparatus and sensory worlds allows us to put our sensory apparatus and sensory world into a wider perspective and context.

\section{Animal Senses}

One of the most neglected areas in the philosophy of perception concerns animal senses. It is surprising how many philosophers write about perception in the apparent belief that humans are the only perceivers in the world. Human senses evolved through the same natural process as other animal senses, so there is no reason to regard human senses as special, or better than, other animal senses. All living organisms, both animal and plant, have some ability to perceive the world around them. Plants are able to detect and turn towards sunlight and flowers and leaves will open and close depending on weather conditions. Animals obviously have vastly more complex sensory apparatus than plants.

Some animals have sensory apparatus that have no human counterpart. Some seem to be able to detect vibrations in the Earth giving rise to beliefs that some animals are able to predict earthquakes. Bird migrations occur under particular weather conditions probably because birds are able to measure atmospheric pressure. Some snakes have heat sensors connected to the part of the brain that deals with vision which enables them to produce a crude image showing the presence of warm blooded prey. Some animals such as the water strider can detect ripples on the surface of apparently still ponds. This enables them to locate prey on the pond surface and to identify mates as the males and females each generate a particular pattern of ripples which the other can recognize. Many animals such as butterflies, bees and birds seem to be sensitive to the Earth's magnetic field and use this to find their way when engaged in long migrations. Whales involved in long migrations also appear to use the Earth's magnetic field to assist in navigation and whale strandings seem to result from this navigation system going wrong. Electrical impulses travel throughout animal's bodies and can travel through water. Many aquatic animals, such as the duckbill platypus and fish have developed the ability to detect other animal's electricity which assists them in finding prey. Some fish actually generate electric fields around themselves and have electroreceptors capable of picking up distortions in the field caused by objects such as other fish. The electro receptors are connected to the part of the brain creating visual images so the fish will probably see objects disturbing the electric field but 
probably as a crude shadowy image. Electric fields are generated in order to catch prey and to communicate with other fish for mating and to assist navigation. Some fish are able to generate enough electricity to stun or kill prey. Many of the senses possessed by other animals are so different from those possessed by humans and other animals that those animals must be considered to be in some sense living in different worlds from each other and from humans.

The most important sense for humans is that of sight and the human sense of sight is better than that possessed by most other animals. Yet humans, and other animals, are able to see only a tiny part of the electromagnetic spectrum. The light we see is only a tiny part of the spectrum consisting of electromagnetic radiation of various wavelengths ranging from radio waves of thousands of miles wavelengths to gamma rays of less than a nanometer. An idea of how the same object, for example a star, can appear different when seen in light of varying wavelengths can be seen in the following websites. (https://science.nasa.gov/science-news/science-at-nasa/1999/features/ast20apr99_1 https://en.wikipedia.org/wiki/Telescope). Humans and animals can see only a small part of this spectrum around 400 to 700 nanometers and different animals can see different parts of the spectrum visible to animals. If we could see a larger part, or a different part of the spectrum, the world we live in would be very different from the world we currently see. The wavelengths of visible light are what produces colors, longer wavelengths appear as reds and oranges; shorter wavelengths appear as greens and blues. Some animals such as pythons and rattlesnakes can see the red end of the visible spectrum only, including colors humans cannot see. Other snakes can see the full visible spectrum including colors at both ends of the visible spectrum that humans cannot see. Some fish can see the full visible spectrum that humans can see and also additional colors humans cannot see at both ends of the spectrum. What an animal sees is determined not just by the part of the spectrum it sees, but also by the type of eye it has and the amount of brain power devoted to vision.

Humans devote a lot of brain power to vision, due to the importance vision has for the human species. This means that the image provided by human sight is quite detailed while other animals with less brain power committed to the sense of sight will receive a less detailed image. Rats see a much blurrier image than humans. See www.ratbehavior.org .

There are a wide variety of types of eyes in the animal world, some very simple that only distinguish between light and darkness, others are much more complex. Some single celled animals have photo-sensitive spots spread throughout the cell; others have a single eye area. Multi-celled microorganisms will often devote whole cells to detect light which may be spread around the organism's body or concentrated in one place. Such organisms can distinguish light from dark but cannot see anything else. Some simple animals have developed cup like depressions to house eye spots, so the animal can detect the direction of the source of light.

The simplest eyes capable of creating an image are those where the opening of the cup like depression is narrowed, the narrower the hole, the clearer the image that is obtained. The nautilus, a marine mollusc, has such an eye, and can produce a clear image of a well-lit scene. If the scene is not well-lit however, the opening must be widened to let in more light and the clarity of the image is reduced. This problem was solved with the development of a lens in the eye which bent the light to produce a clear image, while allowing a large eye opening to let in a lot of light.

All vertebrates and many invertebrates have eyes with lens which operate like a camera. The cornea in the eye bends the light which passes through a hole in the iris, which adjusts automatically to control the amount of light entering the eye. The light then passes through a curved lens which bends the light, which forms an upside down image on the retina. The retina consists of rod and cone shaped cells which contain photosensitive pigments which send a signal along the optic nerve to the brain. The brain turns the image the right way up and adds detail to it. In some of the more primitive 
vertebrates the eye rather than the brain does most of the processing. Nerve cells in the retina of frogs respond to movement to such an extent that frogs can probably only see moving flies and insects, stationary prey being incapable of being seen by frogs. Animals in which the eye does most of the processing, usually invertebrates, see a quite different world from those whose brain does the processing.

Insects usually have a compound eye made up of a number of parts called ommatidia. Each ommatidia, acts as an eye, with a lens which focuses light on layers of light detectors. The compound eye gives far less detail than the camera eye possessed by humans although this does not matter much to insects as important objects for insects are usually close and big. The compound eye does allow insects to clearly focus on everything in their field of vision whereas the camera eye tends to focus on particular areas. Compound eyes, come in different varieties, with daytime, nocturnal and underground insects all having somewhat different compound eyes. The number of ommatidia can vary as can the detailed workings of the ommatidia, so that different insects will acquire different views of the world. Many animals with compound eyes, such as dragonflies, flies and bees, live in a fast moving world with the animals themselves, and their prey and predators often moving at great speed. These animals are often able to process images much faster than humans who can process images at the rate of 60 per second in good light while a fly can process them at 300 per second. A fly watching a motion picture in a cinema would see a succession of still pictures whereas we see a continuous motion picture. A fly would also see electric lights as constantly flickering while we see continuous light.

The amount of detail an animal sees is determined by the size of its eyes and the number of light receptors it has and how densely they are packed together. Humans have 200,000 light receptors per square millimetre providing good resolution while buzzards have 1 million per square millimetre providing them with much better resolution. The light receptors are often concentrated in different areas of the eye in different animals. Birds often have an elongated strip of light receptors to provide detail of the horizon and a circular group of light receptors for seeing straight ahead. In birds of prey the circular group of light receptors are contained in a concave pit which magnifies the image produced by those light receptors. This helps them see potential prey from high in the air.

Predators normally have two eyes in the front of the head which gives them two overlapping views known as binocular vision, which enables them to judge distance, to see greater detail and to have better vision in poor light. Prey, usually have eyes on the side of the head, giving them a wide field of view, often 360 degrees, so they can see potential predators from all directions.

Light receptors come in two types, cones and rods. Cones are responsible for creating color vision. Cones come in three types in humans, dealing with blue, green and red light and these colors are mixed in the brain to produce color images of many hues. Most primates have the same three colors we have but many other animals have two colors so their view of the world is different from ours. The animals with the best color vision are birds which can see five colors and a wide variety of hues that can be made from those five colors.

Some animals, particularly fish, such as goldfish, and insects, such as bees, can see colors we cannot, due to an ability to see colors beyond what humans can see on the electromagnetic spectrum. Goldfish can see both far red and ultra violet light, neither of which we can see, while bees, other insects and birds can see ultraviolet light leading them to see a world of quite different colors than we see.

Sound is caused by vibrations in the air which are converted by our ears and brains into the sounds we hear. The vibrations, or sound waves, vary in frequency, which is the number of times the air vibrates each second. A slow vibration, results in low frequency waves, which produce low pitch 
sounds, while a faster vibration produces higher pitch sounds. Frequency is measured in cycles per second or hertz.

Different animals hear sounds of different frequencies. Humans can usually hear sounds between 20 and 20,000 hertz, although this varies from person to person. Often children can hear sounds higher than 20,000 hertz while the elderly can only hear sounds of up to 8,000 hertz. Larger animals usually hear lower frequency sounds while smaller animals usually hear higher frequency sounds. Elephants can hear sounds from 4 hertz to 18,000 hertz while mice hear sounds from 900 hertz to 90,000 hertz.

All mammals have similar ears but they vary according to each animal's requirements and this affects what each animal hears. Sound waves travel through the ear and press against the membrane of the eardrum which vibrates and the vibrations move along three bones to an oval window. The vibration of the bones amplifies the sound eighteen times for humans and the vibrations pass through the oval window to the cochlea which converts the vibrations into nervous impulses which the brain turns into sounds.

The kangaroo rat, a nocturnal desert animal hunted by owls and rattlesnakes at night, has an extremely large eardrum while its oval window is very small. This causes the vibration to be amplified one hundred times, as opposed to eighteen times for the human ear, and allows the kangaroo rat to hear faint sounds such as air passing over an owl's wings and the movements of rattlesnakes allowing the kangaroo rat to jump away from those predators. Such extreme sensitivity to sound is important in the extremely quiet desert but in a noisier environment it would deafen the animal.

The size and placement of ears can affect what is heard. Some animals such as the jerboa, rabbits, hares, goats and deer have large ears that collect and direct sound into the ear hole. Their ears are also side by side on top of the head and can be turned to better receive sound from any direction. Human ears, on the other hand, are small, on the side of the head and cannot normally be moved and so are not particularly good sound gatherers. However they are good for locating the source of sounds. This is because human ears are about 20 centimetres apart so that the sound reaches one ear a short time before it reaches the other ear. In addition when trying to locate the source of a sound people turn one ear to it so that the head blocks the receipt of sound by the other ear. These factors mean humans are better than most animals at locating the source of sounds.

Frogs ears are sensitive only to the frequency of the calls of other frogs and noises of predators. Unnecessary noises are not heard as the brain only recognises some sounds and not others. Female frogs can hear the calls of male frogs of the same species while remaining deaf to a host of other sounds including the calls of frogs of other species.

Many insects communicate by rubbing their legs together or simply by beating their wings. This produces a sound instantly recognizable by potential mates who head quickly to the source of the sound. Fruit flies come in about 2,000 species and look quite similar but avoid inter breeding as each species has a particular system of wing beats, that produce a unique sound, that can be identified by other members of its species. Predators such as spiders can also hear the sound of an insect's wing beats and can use this to locate prey.

Many birds appear to be able to hear very low frequency sound known as infrasound. Pigeons appear to be able to hear sounds as low as 0.1 hertz, while the lowest frequency humans can hear is 20 hertz. Infrasound can often travel for hundreds of miles allowing birds to hear the sound of breaking waves and the "hum" produced by desert sands of a 1 hertz frequency and of winds passing over distant mountain ranges all of which may act as guides for birds in long distance migrations.

Infrasound calls also seem to have been used to attract mates. Capercaillies, a large grouse, appear to emit low frequency infrasound calls during the mating season which attracts mates through 
kilometres of forest. Elephants also produce infrasound to attract mates and to get help when an individual elephant is in trouble and to warn of danger.

Rodents such as mice and rats often emit ultrasonic calls of up to 100,000 hertz. Young mice in trouble emit calls of 45,000 to 88,000 hertz to attract their mother. High frequency sounds travel only quite short distances as they are easily absorbed by the surrounding environment. This is fortunate for mice as cats can hear ultrasonic sounds of up to 70,000 hertz.

Bats use ultrasonic echolocation to produce a sound picture of objects in front of them. They emit sounds of up to 200,000 hertz which bounce off anything before them and their large ears collect the echoes and send them to the brain which produces the sound picture. Tiny insects can be detected at night from up to 20 metres away by the echo from sounds emitted by the bat in a series of pulses of varying frequencies that can reveal distance and fine detail. Many insects however can detect the bats sound beams and can take evasive action.

The sense of smell is found in just about every animal. Even single celled organisms can detect the smell of their food and move towards it. The difference between smell and taste in many animals is somewhat obscure with some animals having receptors that perform both tasks. All mammals have a sense of smell that works in a similar manner, although the sensitivity to smell varies greatly. Smell operates by air containing chemicals flowing over a membrane of tissue covered by mucus in the nose. The membrane consists of 10 million cells containing tiny hairs and particular areas on the hairs react to particular chemical scents and the overall response of the hairs produces the smells we experience. The human membrane is 10 square centimetres in size, while a dog's can be up to 150 square centimetres. This explains why the human sense of smell is so inferior to that of many other animals.

Smell is often used to detect the presence of predators. Deer and antelope, when eating will periodically raise their heads to sniff the air to check there are no predators around. If danger is detected the animals flee and release scents which communicate the danger to other animals.

Mammals have scent producing glands on various places on their bodies, which they use to send scent messages to other animals. Male mammals often use their scent to warn off other males of the same species. They also use scent to guide them back to their homes and social insects like ants leave trails for other insects to follow to sources of food.

Some animals such as salmon and some birds appear to use smell to assist them when on long distance migrations. Salmon born in a particular stream will migrate hundreds of miles to the oceans and several years later will return to the stream they were born in. They probably use the sun and a magnetic sense to help find the birth stream but it is likely that smell is also involved as the chemical make-up of both sea and fresh water varies in different places and experiments have shown that salmon can detect chemicals in the water and use them to navigate. Migrating birds are also believed to use smells carried on winds as guides in long distance migrations.

Smell is used extensively in the animal world for mating purposes. Male dogs can detect bitches on heat from some distance and vixens attract foxes through the sense of smell. Insects can send long range mating scents. Female emperor moths release scents which drift downwind and the males sense of smell is so good it can detect the female from 5 kilometres distance.

Termite colonies are controlled by smells. The queen's scent runs through the nest and causes the workers to feed and groom her and stops them from producing ovaries. If the queen dies the workers produce eggs and build cells for a new queen. If there is an imbalance in the particular types of termites, for example, if many soldiers die, the decline in the odour produced by soldiers causes young termites to become soldiers to replace those who have died. 
Our brief study of animal senses shows that animal senses are directed to helping animals find food, survive predators, find mates, and for navigation. They are not designed to give animals an objective and accurate view of the world but to ensure the survival of the individual animal and of its species. The animal's senses are geared to particular parts of the electromagnetic spectrum, to particular audio frequencies, and to certain chemical stimuli which create an environment within which particular species live their lives. They have no awareness of sights outside their portion of the electromagnetic spectrum, of sounds outside the frequencies they can hear and of chemical stimuli beyond their ability to smell. Yet such sights, sounds and smells, beyond their sensory apparatus, are every bit as real as those they are aware of. Each species lives in its own sensory world, or ecological niche, with a particular range of sensory stimuli available to it and are quite unaware of what happens outside its world.

The human sensory apparatus is directed towards a particular ecological niche. It needs energy to run and it does not provide us with any more information than is necessary for the survival of the individual human and of the species. It is good at telling us whether there is a tree in front of us and there will be wide agreement among humans about such matters. The human sensory apparatus is not directed to providing us with information about the universe as a whole or as to what is the best political, religious or economic system, which is why we often seem to disagree on such matters.

There seems to be no reason why the human sensory apparatus should be considered better, more reliable or truer than those of any other species. The human sense of vision is better than that of most animals, but it is not the best amongst animals as many birds have a much better sense of sight. Human hearing is reasonably good but many animals have better hearing while the human sense of smell is amongst the worst in the animal world. Just like all the other animals, humans live in a particular sensory world and until the development of modern science had little idea of the sensory world of other animals. Even now our knowledge of the sensory worlds of other animals is quite limited. We still don't see what they see, hear what they hear or smell what they smell. The situation is quite similar to that mentioned by Howard Hughes in Sensory Exotica (Hughes, 1999, 1) when he refers to a story concerning four blind Indians and an elephant:

"One day they went to visit an elephant for the first time. The first stepped towards the animal and happened to touch its ear. The elephant is much like a fan he concluded. The second touched the leg and though the first, a fool. Elephants are nothing like a fan he said. They are more like a tree trunk. The third felt the elephant's side and concluded elephants are like walls. The fourth touched the trunk, and decided elephants are like large snakes. The message, of course, is that we should not mistake the way things seem to us for the way they really are."

Not only do we miss out on a lot of what is happening but often the same thing will appear differently to members of different species. The compound eye will give a different view of a particular thing, than is provided by the camera lens eye, we have. Many animals have lens in their eyes that allow them to focus at different distances. This ability varies with different animals so different animals acquire different views of the world. The same sound may be heard quite differently by different species or might not be heard at all. Amplification of sounds occurs to varying extents in different species ears. A flower may smell quite differently to humans than it does to bees or other insects. A bad smell to one animal can be a nice smell to another.

If you combine the effects of different species having different types of eyes, different positioning of eyes, seeing different parts of the electro-magnetic spectrum, seeing with different degrees of clarity and blurriness, processing images at different speeds and seeing different colors due 
to different cones in the eyes, each species capable of vision, will see the world very differently from other species. If you combine the effects of different species hearing at different sound frequencies, having varying degrees of sound amplification and different abilities to recognise the sources of sound, each species hears differently from other species. If you combine the effects of different species ability to detect different smells and to smell the same thing in different ways, each species will perceive different smells from other species. Each species senses of touch and taste also vary from that of other species and many species can detect the Earth's magnetic field and electric fields generated by other animals. Each species will have certain images, sounds, smells and other sensory stimuli available only to that species and which will be unknown to other species. It is apparent that each species lives in its own sensory world. This sensory world is the ecological niche within which each species lives and each species sensory apparatus is directed not at providing information about the external world, but at the species effective functioning within the ecological niche.

Our sensory apparatus is good at telling us if there is a tiger in front of us or whether a piece of fruit is fit to eat. It is not very good at telling us whether a god or gods exist, what is a good political system, what is the best economic system, whether there is an after-life, what are the basic constituents of matter, understanding the movement of celestial bodies and about the appropriate relationship with other animals. There is little agreement on such matters and human beings have even been known to kill each other when in dispute over these matters.

Philosophers have a habit of debating the reliability of sense perceptions; they would do better to ask, when is sense perception reliable and when is it not reliable? The ultimate source of our disagreements over politics, science, economics and religion lie with our sense perceptions. Science, religion and politics are not directly known compared to our knowledge of the existence of tigers, fruit, trees, tables and people. The human sensory apparatus works better when it deals with things directly related to the species survival.

There are millions of different species on this planet. Each species will see the same thing in different ways or may not see it at all. This suggests there is no single, true, real view of what is being observed and no particular form in which, what is being observed, can exist in when it is not being observed. Each of the many different views, different species can have, can only come into existence when an observation is made. Before the observation is made, the particular view seen by an observer is not seen by anyone, so it does not exist. The particular view will continue only for so long as an observer keeps observing. Once the observation stops the particular view will disappear as no other being will see the particular point in space-time in that particular way. This means that things will only come into existence when they are observed and must acquire their properties, such as shape, size and colour, only when they are observed. If something existed independently of an observation, in what form does it exist? The form in which a human perceives it, or the form an antelope, dog, bat, snake, bird, frog or insect perceives it? All these animals will see a particular thing in a variety of different ways and some may not perceive it at all.

If something exists independently of observation, does it have the colors that some animals will see it possessing or colors other animals will see it having, or something in black and white as other animals will perceive it? Surely a thing cannot be of one set of colors and another set of colors and black and white at the same time. The only situation where something can be of alternative sets of colors, or black and white, at the same time, is where it is observed at that time by different observers whose sensory apparatus will give it different appearances. But if there are no observers and no sensory apparatus you cannot have something being of different colors or black and white at the same time. So it is hard to see how anything can exist unless it is observed. What you see may well exist in 
some form when not observed, but it certainly does not exist in the form in which you see it, as that form is created by your sensory apparatus, and if the sensory apparatus is not working then, what you see ceases to exist.

The argument given in the previous paragraph is very strong so I will restate it in a slightly different form. Some animals can see only in black and white, so everything is a shade of grey, others can see in various colors. If you cease to look at something and it continues to exist, what color does it have? The color one species sees it in, or the color another species sees it in? Clearly it can't be grey and have another color at the same time. The same applies to other sense perceptions, a vibration in the air sounds different to different species; the same odour can smell differently to different species. Remove the observer, what sound or smell continues to exist? The way one observer hears the sound or the way the other observer hears it? Does an odour continue to exist the way one observer smells it or the way another smells it? Something cannot be of different colors, smells or sounds at the same time. Clearly the sense perceptions a particular observer has disappear when the observer ceases to be making an observation.

Such a view, that things only exist when observed, is totally counter intuitive. Some would say it is contrary to common sense. But common sense tells us the Earth is not moving and the Sun orbits the Earth. It is an examination of the facts and logical reasoning from the facts that tells us the Earth spins on its axis and the Earth and other planets orbit the Sun. Equally, an examination of the facts and logical reasoning from the facts tells us that things do not exist unless they are observed.

The problem is most people have a human centric view of the world and think everything exists in the form humans perceive it and continues to exist in that form when there is no human observer. But there are a million other views every bit as valid as ours. Why should something continue to exist in the form a human sees it and not in the form other species see it? There seems to be no way we can justify a belief that the human view of the world is so special that it continues when there is no observer, while other species views of the world disappear.

When we say things don't exist unless observed, it means they don't exist in the human sensory world. They may exist in other species worlds and possibly in other forms quite different from any species world. Our normal ideas of existence are too simplistic; there are multiple ways in which things can exist and multiple worlds they can exist in. The human sensory world is only one such world. Tables, trees and people only exist in the human sensory world when observed with the human sensory apparatus.

Each species has its own sensory world, which are often very different from each other's sensory world. There is some overlap between these worlds but there are many aspects of one species world which will be completely unknown to members of other species. The human view of the world is only one view and is no more valid than that of any other species.

A significant part of the history of science has been the dethroning of human beings from being the centre of existence. Copernicus and Kepler showed the Earth was not the centre of the universe with everything orbiting the Earth. Darwin showed that the human being is just another animal, which has evolved like all other animals and shares ancestors with all other living species. It is time to dethrone the human view of the world and recognise that it is just another view of the world, no more real or true than the view of any other species.

It cannot be argued that the human ability for logical analysis or rationality allows us to know the real world as it is, because all our logical analysis is based upon information derived from our senses. It is only logical analysis about what is happening in our sensory world, not logical analysis about how the universe really is. Sense perception takes place prior to logical analysis so the logical analysis must be based on the sense perceptions. An intelligent dogs smell based explanation of the 
universe would be as good a description of the universe as is general relativity. But neither explanation is an explanation of "reality". They are merely explanations of a set of sense perceptions.

In addition humans are not that rational and many animals seem to have some degree of rationality. Bertrand Russell in The Problems of Philosophy (Russell, 1912, 34-35) notes that animals use induction, the belief that past examples will continue into the future. If, you are nice to a cat, feed it and pat it, it will assume you will continue to be nice to it. There is the famous example of the inductive turkey expecting to be feed, as he was many time before, getting killed on thanksgiving. We are in the same position as other animals. The problem of induction applies just as much to humans as animals. Induction is an important part of modern science, without it modern science would not be possible. Many animals are also capable of making and using tools, making nests and communicating by means of sounds. The conclusion seems to be that while humans are better at logic and rationality than other animals, it is only a matter of degree, not an absolute. Other animals are also capable of logical behaviour and understanding. We and the other animals are on a continuum of rationality and it is an example of a false dichotomy to suggest humans are totally rational (they are not) and other animals have no rationality (they are capable of a degree of rationality). There is no absolute divide between humans and other animals when it comes to rationality. It is a relativistic situation rather than an absolute divide. We tend to draw absolute dividing lines between us and other animals but often the differences are a matter of degree rather than absolutes.

\section{Neurology and cognitive psychology}

One area of science that has made considerable progress in recent years is neurology, the science of the brain. The following study of the brain relates to the human brain, but with suitable modifications would apply to the brains of many other animals. The human brain is made up of between 100 billion and 1,000 billion cells called neurons. Neurons are connected to other neurons; a neuron might have between 100 to 10,000 connections with other neurons. There are two types of connections, dendrites which handle input to the neuron and axons which deal with output from the neuron. Synapses within dendrites and axons release chemical substances which spread to nearby neurons and cause them to react in certain ways.

The brain consists of a number of different areas, each of which handles, sometimes in combination with other areas, specific activities. The area handling vision is the occipital area which is located at the rear of the brain. This area is divided into a number of sub-regions known as v1, v2, $\mathrm{v} 3, \mathrm{v} 4$ and v5 (v stands for vision). Visual information arrives at the area v1 and then is sent to v2 where it is separated into various parts and sent to v3, which deals with form, v4 which deals with colors and v5 which deals with movement. Other adjacent areas of the brain are involved in analysing what an object is and where it is. The other senses also have particular areas of the brain where sensory information is processed. The frontal lobes deal with movement and smell and the temporal lobes with sound.

The brain starts to form in humans about two weeks after conception. A neural plate begins to develop which then forms into a tube which then develops into the brain and spinal cord. The brain grows in size as cells which eventually turn into neurons divide into two and move towards particular areas of the brain. Eventually at birth the brain is about 350 cubic centimetres in size and after birth it continues to grow to about 1400 cubic centimetres at around 4 years of age. Nine months after conception most of the neurons have moved to the appropriate brain areas. When in place each neuron 
will begin communicating with other neurons by establishing synaptic networks with other neurons. Axons and dendrites will be sent out and will establish connections with other neurons. There will be a considerable genetic influence on the connections made between neurons, but the environment and personal experience of the individual will also have a considerable effect on the connections established between neurons. Environmental influences are shown in that if an area is not used for its normal functions it is likely to be taken over and used for other purposes. An example of this is where a baby's eye was bandaged for two weeks due to a minor infection. This was at a crucial time for the formation of connections between the eye and the neurons in the area of the brain dealing with sight. As the eye was not being used connections were made by nerves from the other eye with neurons that would normally be connected to the bandaged eye with the result that the baby permanently lost the use of the bandaged eye. The environment however does not just cause unused abilities to be lost. If a particular activity is extensively carried out, the neurons involved in that activity will establish more and more connections, which will improve the ability to carry out the activity. The neurons are very reactive to whatever is happening to the individual in the outside world, forming and abandoning connections in relation to how much use is made of them. If a neuron is insufficiently used it will die while neurons actively used will establish more and more connections. The more connections that are established by neurons involved in particular activities, the better the individual can carry out the activities.

The brain stops growing around the age of 16 and the process of forming and losing neuronal connections continues after that age, but at a reduced intensity. The process of forming and losing neuron connections in response to our environment gives each individual a unique brain. By the time a person has reached seventy years of age there is a 5\% loss in brain weight, while by the age of ninety, there is a $20 \%$ loss in brain weight. As people age, neurons are lost, possibly as many as 200,000 per day, and the brain loses neuronal branches and neurons shrink in size. Different individuals lose brain cells from different areas of the brain, so that the symptoms of brain cell death are different for different people, you might lose some here I might lose some elsewhere, so our brains are different and we end up seeing the world and reacting to it in different ways.

As you grow up and have experiences your neuron connections grow in a particular way, to produce a unique brain as everyone has different experiences. We interpret what we see and hear not just based on what is directly heard and seen but also in relation to our previous experiences contained in our memory. If you are eating something and have an unpleasant experience that can make you dislike that food. Bad experiences when listening to music can make you dislike that song or piece of music. The effects of experience are to modify, not so much the number of neurons, but rather the connections between them.

A wide variety of experiences may affect the connections between neurons. Playing sports such as cricket and baseball will result in an experienced player seeing the ball differently from a person who has never played the game before. An experienced player will see a fast ball because their brain has been trained to watch for it, while an inexperienced player may not even see the ball. The inexperienced player will not know what to watch for, where to focus and their brain is just not trained (i.e. has insufficient neuronal connections) to see a ball that fast. An experienced chess player will see a position in a chess game, and quickly see a range of possible moves, while an inexperienced player will not see those moves. Judges in ball-room dancing competitions will see many things in the competitor's performances that non experts will not see. The judges will know what to focus on and what is considered important when judging the competitors.

Human beings and almost certainly many other animals do not just experience shapes, colors, sounds and other sensations; they also react emotionally to their experiences. The process by which 
people react emotionally to sensory information seems to involve the information being associated with particular emotions such as fear, anger, sadness, good or bad due to a neurotransmitter called dopamine being sent to an area of the brain known as the amygdala. The amygdala assigns a particular emotion or emotions to the sensory information which provides our emotional reaction to our sensory experiences. Different people react differently to the same sensory information due to the different emotions being associated with the information from the amygdala. Two people might see a dog and one might feel attracted to the dog and wish to pat it, the other may feel fear and want to get away from the dog. Which reaction a person has will depend on prior experiences of dogs which will result in different wiring in the brain.

Human beings are also able to read the emotions of other people and feel the emotions of other people. However, different people may read facial expressions differently. If angry expressions have been followed by violence in a person's prior experience that person will feel different emotions when confronted with angry expressions than a person who had experienced angry facial expressions that had not been followed by violence. Some individuals for example those with Asperger's Syndrome have a much lesser ability to understand facial expressions than those without Asperger's.

There are a number of circumstances which illustrate how changes in a person's brain can affect what they see. The autoscopic delusion or doppelganger effect involves a person seeing another version of his or her self. This is considered to occur when parts of the brain dealing with vision and those dealing with information about our own body interact to produce a vision of something we would normally feel. Some people experience synaesthesia, a condition in which there is a mixing of sensory perceptions so that they may taste colors or hear colors. Research has suggested that this may be caused by the electrical impulses stimulated by a person seeing a color, travelling not just to the area of the brain dealing with colour, but also to those dealing with tastes or sounds. This may be caused by genes producing unusual connections within the brain dealing with tastes or sounds. This suggests there may be no real colors, just routes for processing stimuli which if they go to one area of the brain produce certain sense perceptions and if they go to another will produce other sense perceptions. The phenomena of phantom limbs where a patient still feels the presence of a limb after it has been amputated suggests the importance of the brain in perception. The limb may be gone, but the patient still feels its presence simply due to brain activity. Some people have brain disorders that result in them being unable to recognise familiar objects or faces. These disorders known as agnosia and prosopagnosia seem to result from damage to particular parts of the brain where the awareness of what an object is, or whose face is seen, is stored. However recognition of objects and faces occurs in different parts of the brain for different people. A musician will have recognition areas for musical instruments in a different place from a non-musician so the same damage would have different effects on different people's brains. A person not suffering from prosopagnosia may still have difficulty in recognising a person's face if they have not seen the person for a while as neuronal connections for the memory of that particular face will die and the memory and the ability to recognise the face will disappear.

The cases of autoscopic delusion, synaesthesia, agnosia and prosopagnosia can all be regarded as brain disorders caused by a brain damaged in some way. However they still illustrate the crucial role the brain has in what we perceive. Change the brain in some way then what we see, hear, smell, taste and feel will change. The same situation however will exist where the brain is not damaged. The neuronal connections in the brain are constantly changing in response to each individual's personal experiences. John Dowling in Creating Mind states: 
"Our visual system constructs images based not only on incoming sensory information but also on experience. What we see depends very much on our expectations-what we have seen before-as well as on information from our retinas. As noted earlier, vision is reconstructive and creative. The visual information coming from our eyes is imperfect; we construct a logical image based on incoming information and visual memories. If the visual information arriving by way of the optic nerve is ambiguous, we make a logical percept.” (Dowling, 1998, 184)

Each human being has different experiences and memories so each will create different images from the sensory information they receive and from their memories. The ever continuous accumulation of new experiences means that no two brains are the same and no two brains will see the world exactly the same way. In The Human Brain Robert Winston states:

"differences in the way our brains receive and assess incoming sense information create differences in the way we each see the world. In fact, it might be more accurate to speak of individual sense worlds rather than some objective world." (Winston, 2003, 125)

It may be suggested that even though different people have their brains wired differently, they still often see and hear the same things. Two people watching television will see and hear the same things and drivers will normally all see traffic lights as either red or green. However there will still be subtle differences in what two people watching television together will see. They might hear the sounds at slightly different volumes and they might notice slightly different things on the television. At the traffic lights, while two people may see the same colors they may well see different levels of brightness and different shades of the same colour. The two individuals involved may believe they are seeing the same thing on television or at the traffic lights, but if we could directly compare a picture of what each person saw there would be many subtle differences. The extent to which the two individuals see and hear the same thing while watching television and at the traffic lights is a result of the similarities in their sensory apparatus. However no two individuals have exactly identical sensory apparatus and due to this there will always be subtle differences in what they see and hear even if they appear to be watching and listening to the same thing.

If, as Robert Winston suggests, different people see the world in different ways, so as to create individual sense worlds, this may be confirmed by experiments. There is one major problem with this as we do not have direct access to people's sense perceptions to compare them. We are necessarily reliant on people's reports of their sense perceptions and between the sense perceptions and the reports there is much that could produce variable reports of perceptions of the same scene, such as memories fading. This problem can however be reduced by obtaining reports immediately after people have viewed the scene. This area has been much researched in cognitive psychology due to concerns over the accuracy of eyewitness reports in court proceedings. Numerous studies have been made that show that eyewitness reports are likely to be distorted both by an individual's prior knowledge and expectations and by information received after the event perceived. Many of these studies have been made in relation to legal proceedings and it is considered that many innocent people are wrongly convicted each year due to mistaken identification and eyewitness errors. In many cases people convicted by eyewitness testimony had been later cleared by DNA evidence. It has been suggested that eyewitness evidence has caused more wrongful convictions than all other causes combined.

Many studies involve participants being shown pictures or videos of a particular scene and being provided with prior or subsequent additional information, some of which was correct and some 
incorrect, in order to access the effect of the additional information on participants memories. While these studies are mainly aimed at showing the effect of the additional information on participant's memories, they can also show how different people viewing the same scene will provide varying reports of the scene. One study for example, showed a car accident involving a pedestrian and when questioned about the road sign in the scene $75 \%$ correctly answered as to the type of sign but $25 \%$ got it wrong. In another study a video of a scene in which a wallet was stolen was shown to participants who were supplied with additional information both before and after the video was seen. When the participants were asked questions about what they had seen, the answers revealed the additional information supplied before and after the viewing had a considerable effect on the answers. The results however also revealed that regardless of whether or not prior or subsequent information was provided participant's answers to questions of what they had seen varied considerably. It was also noted that $41.1 \%$ of questions relating to the central aspects of the scene were correctly answered while only $23.3 \%$ of questions related to more peripheral aspects were answered correctly. (Scheer, Jennifer B, The Reliability of Eyewitness reports: the Effect of Accurate and Inaccurate Information on Memory and Bias, Colgate University Journal of the Sciences, 119.)

A further set of experiments concerning change detection and change blindness involve scenes in which still pictures, motion pictures and real life situations are changed in front of people often without the change being noticed. Failure to notice the changes occurs much more often in relation to peripheral objects than objects considered to be the centre of interest in the scenes. This suggests attention may be an important factor in change detection with changes in objects upon which the observer's attention is focussed being much more noticeable than changes in objects not attended upon. However observers often failed to notice changes when their attention was focussed on the object that was changed. This suggested something more than attention was required for an accurate representation of an object's details. In an experiment involving substituting a person during a conversation many people failed to notice the change, but those who did notice the change were substantially from the same age group as the individuals who were substitutes. It was suggested people of the same age or social group as the substituted persons tended to make an effort to notice features which would differentiate them from other members of the social or age group, while those outside the group did not put the same effort into observing the details of the substituted persons. Successful change detection requires more than just having one's attention directed towards an object; it requires an effort in noticing the features that were different between the substituted objects. If that effort is not made the change will often not be noticed, but the effort is far more likely to be made if the observer is a member of the same age or social group as the substituted person. What people see depends upon what their attention is directed at, but also in how much effort they make to notice the details of the object their attention is directed at. Different people looking at the same scene may see quite different things, depending on what they direct their attention to and also in how much effort they make to observe the details of the object their attention is directed to. (Failure to Detect Change to People during a Real World Interaction by Daniel J Simons and Daniel T Levin in Cognitive Psychology: Key Readings in Cognition ed by David A Balota and Elizabeth J Marsh (2004) Psychology Press, New York and Hove).

The research made in cognitive psychology would appear to confirm the idea from neurology that different individuals will see the world differently. Each individual brain is wired differently so they will obtain their own individual view of the world. This view will usually be similar to that of other people, but never identical to that of other people. Different people viewing the same scene will give their attention to different aspects of the scene and will make varying efforts at noting details of 
the scene. Experiments in cognitive psychology confirm that different people observing the same scene will not obtain the same view of that scene.

\section{Perceptual relativity}

It is quite apparent that our sense perceptions do not provide us with a single objective view of the world. However, we may get some idea of what the universe is like by imagining what the world would be like if our sense organs were different or if we had no sense organs at all. Let us imagine first a person born with no sense organs or sensory apparatus. What would such a person be like? Obviously such a person would have no sense perceptions nor would he or she be capable of any sort of language. This person would not be able to think, as our thoughts are in words, and would be incapable of imagining anything, because with no sense perceptions, there would be nothing to base any images on. How could you imagine colors and shapes if you have never seen any colors and shapes? Any form of mathematics, whether pure maths or applied maths, would be impossible, as such a person would have no conception of space or numbers. To such a person there is no world, nothing exists; there is no awareness of anything, including self-awareness.

Let us now suppose our person could see, but had no other sense perception although his or her sight was exceptional. Such a person could perhaps see distant planets such as Uranus and Neptune and microscopic life forms invisible to the normal unaided human eye. It could be argued that such a person lives in a different world from normal humans as that person is able to see things other humans are not directly able to see, while being quite unaware of all the sounds, tastes, smells and touch sensations other humans take for granted.

Let us now consider a being with none of the usual human senses, but with the ability to perceive; gases contained in the atmosphere on earth and the ability to differentiate between those gases; infra-red radiation, thus giving the ability to detect objects with a temperature above absolute zero; echoes of sounds sent by the being and bouncing off certain objects, similar to that possessed by bats. If such a being existed, its world would consist of certain colors perceived from objects emitting infra-red radiation, echoes and an awareness of certain gases. Such a being could live on the planet Earth and yet be, in a sense, living in another world from the humans on the same planet. It would perceive things in this world that humans do not see, it would fail to perceive things which humans perceive and would perceive some things in one way that humans see in a totally different way. Yet such a being could live amongst us and view the world quite differently from human beings. There seems to be no reason why such a being's view of the world should be regarded as being less real than those of human beings. To treat human sense perceptions as special, or better, than those of other species is simply a form of human centricity that seems impossible to justify.

There are possibly an infinite number of thought experiments that could be dreamt up involving different sensory apparatus and the sensory worlds they could create. These worlds don't actually exist compared to the actual worlds created by conscious beings whenever they make an observation. However, the development of modern scientific instruments, such as telescopes and microscopes, does allow the creation of a range of artificial sensory apparatus and the sensory worlds they can create.

A further illustration of our world being observer dependent can be seen in the old question if a tree falls in a forest and there is no one there to hear it, does it make a sound? The answer to this is, no it does not. This is because a tree falling in the forest will make vibrations in the air, but vibrations in the air are not sound. Vibrations in the air only get converted into sound by the mechanism and processes of the human (or some other) ear and brain and if there is no ear and brain to carry out this process, the falling tree will make no sound. Sound requires both vibrations in the air and a hearing 
apparatus (ears and brains) for it to be heard. Remove the hearing apparatus and you only have vibrations in the air and vibrations in the air are not sound.

This really comes down to an argument over semantics. If sound is regarded as vibrations in the air, then a tree falling in a forest with no one around, will make a sound. If sound is regarded as something that is heard, then a tree falling in a forest with no one around, will not make a sound. I think sound is best defined as something that is heard as that is what we normally mean by sound.

The same situation exists with the sense of sight. If light is reflected off an object and there is no one there to observe the reflected light, can it be said that the reflected light amounts to an image, such as an observer would see? Again the answer is no. This is because what we see is not light reflected off an object but an image created by the eye and brain of the observer using the reflected light. When light is reflected off an object it passes through the cornea which bends the light waves. The amount of light that enters the eye is controlled by the iris which allows more light in when there is insufficient light and less when there is too much light. Then the light passes through a lens which focuses an image on the retina. The retina contains receptor cells that change the physical energy of a light wave (or light particles) into neural energy which the brain interprets as seeing. The receptor cells, called rods and cones, receive light stimuli and send impulses along the optic nerve to the area of the brain that deals with sight. Here the impulses are converted into the images that we see. Just as with sound, the raw data that enters the eye, reflected light, goes through such a process of change when it enters the human (and other animals) sense organs and brains that it is not at all clear that the images we receive bare any more relation to the reflected light that enters our eyes than vibrations in the air have to sound. The human brain is inadequate to take note of all the stimuli the body senses. It is estimated a brain the size of a cubic light year would be required to process all the information received by the eyes alone. A human being must therefore ignore the majority of the stimuli he or she senses and in turn these stimuli vie for attention. No one has actually "seen" reflected light. All we know about it is what the physicists tell us, for example, that it consists of particles called photons, or of waves, or both. What we see are images processed out of reflected light that may have no more relation to reflected light than a cow has to a hamburger. Furthermore if one removes the observer all that will remain will be the reflected light as the images the observer would normally see will disappear with him or her as there will no longer be any mechanism available to convert the reflected light into the images an observer might see.

It is necessary to distinguish between perceived and unperceived reality when considering perceived and unperceived perceptual stimuli. The distinction between perceived and unperceived reality can be seen if one traces what happens to observed and unobserved reflected light. We could imagine a beam of light striking an object and then being reflected off into empty space. No observation is made of the reflected light so the object is never observed. In this situation the object is never part of a perceived reality, but it may be part of an unperceived reality although this unperceived reality is not known to us. This is the sense in which we mean objects do not exist unless they are perceived; they do not exist as part of a perceived reality. Next imagine a beam of light reflecting off an object and entering the retina of being A who sees the same colors, sizes and shapes as a human. This brings the object into existence as part of a perceived reality and in particular to the perceived reality of being A. Next imagine a beam of light reflecting of the same object and entering the retina of being $\mathrm{B}$ who is unable to perceive colors and who is also unable to clearly differentiate boundaries with any precision and simply sees things in shades of grey that gradually merge into each other, so the boundaries between things are indistinguishable. This would bring the object into existence as part of the perceived reality of being B. However B's perceived reality will not be the same as A's perceived reality.

In these circumstances, the object will not be part of any perceived reality until observed. When observed it will take what form the observer's sensory apparatus is able to give it. Being A will 
see it in one form; being B, will see it in another form. In other words it is indeterminate until an observation has been made. The observation both brings the object into existence in a perceived reality and gives it its particular form.

Our senses have evolved over millions of years in order to help us to survive. They give us information as to whether food is safe to eat, where potential prey may be, whether potential predators are around and where mates can be found. They are designed to give us information relevant to our survival. Information not relevant to our survival, will not normally be available to us. Our senses are not designed to give us an accurate objective view of the world. They require a certain amount of energy to operate and human survival requires that energy is not wasted in providing us with information not relevant to our continued survival as a species. It is hardly surprising our senses do not give an accurate or objective view of the world. They are simply not intended for that purpose.

The problems disclosed by Russell, Special Relativity, the Doppler Effect, other animal perception and by neurology and cognitive psychology, often called the problem of perceptual relativity, have been the subject of much philosophical discussion. Perceptual relativity is how the world looks different to different observers and even to the same observer in different locations or moving in different speeds or directions. Traditionally philosophers have tended to see perceptual relativity in terms of sticks looking bent in glasses of water, or cold hands put in warm water feeling warmer. Perceptual relativity needs to be seen in much wider terms involving animal senses, neurology, cognitive psychology, astronomy, special and general relativity and quantum physics. Perceptual relativity may be a problem, but it is also a clue, or even a key to unlock the fundamental nature of the universe.

Sceptical philosophies based upon the facts of perceptual relativity have a reputation for knocking down philosophical schemes as to the nature of the universe. There is however no reason why the facts of perceptual relativity could not be used as a basis for a philosophical scheme as to the nature of the universe. The process of identifying the facts and reasoning from the facts are themselves subject to perceptual relativity, so it is impossible to be certain of them and impossible to be certain of any philosophy of the nature of the universe derived from them. It does however, have the virtue of an honest acceptance of the limitations of human knowledge and does not involve any claim to a priori knowledge, which humans simply do not have. Such a philosophical scheme is presented in Part II of this paper. Philosophies built upon a priori knowledge invariably fail as there is no a priori knowledge. Even pure mathematics requires prior perception as there can be no concept of number, for example of plurality or multiplicity, or of shape without some initial perception. A priori knowledge is a philosopher's fantasy and is as real as unicorns and leprechauns.

George Berkeley suggested that the problem of perceptual relativity, if it is a problem, showed the table examined by Russell did not really exist in any objective state and there was no such thing as matter. (Berkeley uses the term "matter" in a quite different way than it would be used by modern physicists). All that could be said to exist were sense perceptions which vary according to which observer is making the observation, the position of the observer and the circumstances of the observation (such as the amount of light). Such a variety of contradictory sense perceptions (the table cannot be of different colors, sizes and shapes at the same time) suggests there could not be a real table there but only a set of sense perceptions with no continuing identity. Berkeley suggested that these sense perceptions existed only in the mind. They were mental entities only and could only exist when an observation is made. He considered "To be, is to be perceived" and that no world existed beyond our sense perceptions. 
However the Berkeley interpretation is not the only possible interpretation of perceptual relativity. If one looks at the problems shown above it would appear that what we see varies with the sensory apparatus used to make the observation and the conditions within which the observation is made. If one changes the position of the observer, the shape and colour of things change; if a different sensory apparatus is used, such as another animal's sensory apparatus, there may not be any colors at all or there may be additional colors that humans are not aware of. Sounds, smells, taste and touch vary with the sensory apparatus and the conditions under which they operate. Sounds are vibrations in the air, but if there is no air, for example in outer space, no sounds can be heard. The ability of dogs to detect smells and sounds not perceivable by humans shows that different sensory apparatus will produce different sensations. Tastes appear to vary from person to person and from species to species. What is poisonous to one species may be food for another. Touch varies with the conditions available, for example a cold hand placed in cold (but less cold than the hand) water, will feel warm. The feelings obtained by touch also vary with the part of the body used for the touch as some parts of the body are more sensitive than others. If the sensory apparatus or the conditions of the observation are changed, then different sensations will be produced. This suggests the sensory process involves a relationship between the sensory apparatus, the conditions of observation and whatever, if anything, is out there. If any of these are altered different sensations will be produced. There seems no basis on which we can claim any of these sensations are more real than any other of the sensations.

This suggests that Berkeley's interpretation may be wrong in some respects. One is that he seems to go beyond what can be inferred by the facts of perception. He does this when he claims there is no such thing as matter and that sense perceptions are mental entities only. In order to claim there is no such thing as matter, it would be necessary in some way to go beyond our sense perceptions to see whether matter exists or not. This, known as going beyond the veil of perception, is something we are not able to do. The appropriate attitude to matter is agnosticism, rather than atheism. Equally the claim that what we see are only mental entities is doubtful, when what we see seems to involve a relationship between the sensory apparatus, the conditions of observation and whatever, if anything is out there.

What is observed when an observation takes place is sense perceptions or sensations. Given that the sensations change if different sensory apparatus are used to make the observations, this suggests there is no single objective reality available to us. It may be that such a reality exists, but we can never know it as our sensory apparatus does not give us any special privileged way of observing the world that allows us to get beyond the sensations. We have no idea what is beyond the veil of perception. Berkeley suggested there was nothing, while Kant suggested there was "noumena" or the "thing in itself" and simply stressed the unknowability of the noumena. In effect Berkeley claimed matter did not exist (i.e. nothing beyond the veil of perception) an ontological matter, while Kant considered it to be an epistemological matter i.e. we have no way of knowing what is beyond the veil of perception. I think Kant's view is to be preferred as we are not in a position to say there is nothing beyond the veil of perception and the acceptance of an idea such as noumena helps to explain why people with similar sensory apparatus see different things at different times. Without noumena a person's sense perceptions would lack variety.

Sensations will vary if you change any of:

i) the observer or sensory apparatus used; or

ii) the conditions of the observation; or

iii) the point in space and time that is being observed e.g. the observed 
It is because sensations change if you alter the point in space or time that is observed, that a person can see one thing and at another time can see something else. When I refer to a relationship between the observer, the conditions of observation and the observed, I mean you will get different sensations if you alter any of the observer, the conditions of observation or the observed.

This means that if the observer is changed then a new sensory world is created and that world, and the things in it, only come into existence when an observation is made. The observer creates the world that he or she sees by making the observation.

The idea of noumena and our inability to perceive the real world (if there is one) seems consistent with other philosophical theories such as Popper's falsificationism, Hume's analysis of cause and effect, the problem of induction, Poincare's theory of conventionalism and Kuhn's theory of scientific revolutions. These theories all suggest there is something unknown to us, something beyond our sensory perceptions, that we have no access to and is unknowable to us.

Popper suggested a scientific theory could never be proved correct but could only be falsified by observations that contradicted the theory. This is because while we can observe phenomena that contradict the theory, we can never look beyond or behind the theory to check whether it was correct as this would involve observing the noumena which is impossible.

Hume noted that we can never prove cause and effect; all we can do is observe the correlation of phenomena. We can observe one billiard ball hitting another and the second ball being set in motion, but we can never prove the second ball was set in motion by the first. We can never prove a necessary connection between the first ball hitting the second ball and the second ball being set in motion. We can merely show the one event, the first ball hitting the second was followed by the second ball being set in motion. The inability to show the first ball caused the second ball to move is because we can only see the phenomena and we cannot see the noumena. Theories in physics about the transfer of energy from one ball to another are also theories of phenomena. Such theories explain the relationship between phenomena but are unable to go behind the phenomena to prove the theories are correct. All they can show is that the theories involve no internal contradictions or contradiction with well-established phenomena. They cannot however go beyond the phenomena.

The problem of induction is also consistent with the idea of noumena. With induction all we have is repeated examples of the same phenomena in a particular situation, but we can never be certain that in the same situation the phenomena will always be repeated. This is because we are unable to see behind the phenomena to see the cause of the phenomena. This would involve observing the noumena which cannot be done.

Poincare's theory of conventionalism suggests there can be alternative scientific theories in matters such as planetary orbits as a single set of observations can be explained in a variety of ways. Poincare wrote "If a phenomenon admits of a complete mechanical explanation, it will admit of an infinity of others which will account equally well for all of the peculiarities disclosed by the experiment." (The Foundations of Science, Paris, 1892). Planetary orbits can be described as elliptical orbits in three dimensional flat space or as circular orbits in four dimensional curved space-time. By adopting different rules for orbits any structure of space can be used to explain the observations of the planetary orbits. Equally, by varying the structure of space, any description of planetary orbits is consistent with observations of the orbits. There is an infinite number of ways in which planetary orbits and the structure of space can be described all consistent with the observed planetary orbits. Poincare suggested there is no way in which we can be sure of what is the true structure of space or planetary orbits. In order to know these things it would be necessary to go beyond the phenomena and see the noumena which we cannot do. Poincare's observation applies to all scientific theories, not just planetary orbits and the structure of space. 
Kuhn's theory on scientific revolutions considered our theories keep changing because the facts keep changing when new discoveries are made. The constant revision of facts is evidence we are dealing with phenomena. If we were dealing with a single observer independent world the facts would not change, they would be the same all the time.

The reason why humans have difficulty in accepting their world is observer dependent and that different sensory apparatus will produce different sensory worlds is that humans have only one set of sensory apparatus (e.g. the normal human senses) that give a more or less similar and coherent view of the world. As all humans have very similar sensory apparatus they obtain very similar sensations, so that within the species there is a lack of variety in the way the universe is seen. Everyone seeing very similar sensations leads to the belief this is the one and true view of how the universe is. Coherence among our senses is the result of evolution and it has the effect of fooling us into thinking there is only one world, our world. We are unable to use an alternative sensory apparatus that will show different views of the world, except possibly, recently with modern science. The problem is a form of human centrism, the belief that the way humans see the world, is the way the world is and the only way it is or could be. Anthropologists have observed the same phenomena in different cultures around the world. Nearly all cultures believe that their view of the world is the right and only view of the world. They tend to be culturally centrist or as it is often expressed ethno-centric. This was because there was little contact between different cultures, so the people in one culture could agree amongst themselves that their view of the world was correct with little to challenge that view. However, in the modern world, cultural centrism has to some extent broken down and new concepts such as cultural relativism have emerged. This is because we have come to see that there are a considerable number of alternative and in many ways contradictory views of the world and it is often difficult and sometimes impossible to rationally choose between them. What gave rise to cultural relativism was the realization that different people saw the world differently. What gave strength to ethno-centrism is that everybody within a culture tendered to see the world the same way. All human beings have more or less the same sensory apparatus so we all see the world in more or less the same way and we tend to be unaware that there may be other ways of seeing the world. This is a form of species centrism which is similar to cultural centrism.

\section{Arguments against the argument from perceptual relativity}

It is sometimes considered that we see objects rather than sensations and that perceptual relativity does not apply to our perception of objects. A person lacking a word for a particular object can still see, smell, touch and taste the object. It is claimed that perception of objects does not depend upon and is not relative to the perceiver's linguistic, conceptual, cognitive or scientific knowledge. (Audi (ed), 1995, 569). If we see objects and perceptual relativity does not apply to objects, then that enables us to see a single real world, to see the world as it is.

There are a number of problems with this argument. The first is that it fails to consider that perceptual relativity is not based solely upon the human perceiver's linguistic, conceptual, cognitive or scientific systems. It is also based on the sensory apparatus available to non-human perceivers and this may vary widely between such perceivers. Different animals with different sensory apparatus may well perceive as different objects, what we perceive as the same object and what we perceive as the same object, as different objects. It may well be that what I see as a computer keyboard on a table, as two different objects, my cat may not make any distinction between keyboard and table. My cat will not know the keyboard and table have different functions and is not able to examine the keyboard to 
see that it is not attached to the table. Much as I see two separate objects, my cat may regard the keyboard and table as a single object of two different colors. The view that perceptual relativity does not apply to objects is a human-centric view that assumes the human view of the world is in some way special. It is hard to see how this view could be justified.

A further objection to the view that we see objects rather than sensations and perceptual relativity does not apply to objects, is that it is not clear what an object is. There seems to be no objective criteria for deciding whether what is observed is an object. In practice we often make such distinctions based on criteria such as attachment and function. If what we are observing is not attached to anything we often regard it as an object. However even if what we are observing is attached to another thing it may well be regarded as an object, as an object can be made up of other objects. If what we are observing has a particular function then we may well regard it as a separate object. A pen sitting on a table has a particular function, namely writing, so it will be regarded as a separate thing from the table which has a different function. An ashtray sitting on a table will be an object, but what if it is nailed or glued to the table. It would be attached the same way as the tables legs might be attached, but whereas the table's legs would be regarded as part of the table, as well as possibly being objects in their own right, it is not clear whether an ashtray glued to a table would be regarded as part of the table. What if the ashtray was simply a hole cut into the table top, so it is not attached to the table but an integral part of the table, but has a function quite different from the rest of the table?

When I look at my door I see a brown varnished door of different shades of brown corresponding to the grain of the wood. Some parts of the wood are darker than other parts. I also see a brown wooden door handle. Most people would say the different colors of parts of the wood do not constitute separate objects, but the door handle could be considered a separate object from the door. The only way we could say the door handle is a different object from the door is that we know the function of the door handle is different from that of the door and that we know the door handle is screwed on. However knowledge of the function of the door handle and of screws is culturally relative. It is not at all obvious to people unfamiliar with doors and screws that the door handle enables people to open the door and that the door handle is attached to the door. People in a non-door culture would have no reason to think that the brown wooden shape, which we would call a door handle, has any more specific function or any different function than the different colors that constitute the wooden part of the door. They would also have no reason to believe that the door handle was connected to the door and would see it as part of the door as much as the differently coloured parts of the wooden door. The knowledge as to whether a particular item is attached to another item or is part of that item would be culturally relative as it would involve knowledge of how items can be attached, for example glued, nailed or screwed together. Knowledge of function is often necessary to decide whether a particular colour and shape constitutes an object, but knowledge of function will certainly vary across cultures so the perception of whether a particular colour and shape is an object will vary across cultures. If knowledge of function is not necessary to decide whether a particular shape and colour is an object, then even in a particular culture there will often be disagreement as to what is an object.

If function is an important criteria for deciding whether something is an object, there would be many problems with humans from non-modern cultures having the same view as to what is a thing as people in modern societies. Hunter-gatherers and many other non-modern peoples would have no idea what the function is of a vast range of products used in modern societies. This would mean their judgment as to what is an object would be quite different from that of most modern people. An example of this was when Cortez and his Spanish horsemen landed on the coast of Mexico, the native Americans believed his horse riders were a single animal rather than a man on top of a horse. They 
were quite astonished when they saw a man dismount; to them it looked as though the animal was coming apart before their eyes. The Americans thought the horse and rider were a single animal and a single object. The Spanish would have considered the horse and rider was one object on top of another.

This suggests what we regard as an object is a learnt experience, rather than something that is directly known through perception. Our most immediate sensory experiences are sensations rather than objects and we construct the objects from the sensations using our knowledge of what is being observed. What is an object will vary from culture to culture depending on what knowledge is possessed by the culture about that which is being observed.

It is also doubtful that perceptual relativity does not apply to objects. While there may be some doubt as to exactly what is an object, if one chooses a typical object, say, with a total lack of originality, a table, one can say perceptual relativity applies to it. Its colour, shape and size will still vary with the position and motion of the observer and the amount and angle of the light falling upon it. This is because perceptual relativity applies to all of the properties of an object and the object is nothing more than the sum of the properties of the object. If perceptual relativity applies to the properties of an object then it must apply to the object. It is hard to imagine an object without its properties and when we talk about an object all we are talking about is the totality of its properties. Even if objects are something more than the totality of their properties, is that something more, something which will stop perceptual relativity applying to objects?

The whole concept of an object is murky. It is likely that other animals would not have the same view as to what is an object as would human beings. Obviously they will have little idea of the function of many human objects and would also often be unaware of the degree or manner of attachment objects may have to other objects. However even between human cultures what is an object will often not be clear as people from non-modern cultures will have no idea of the function of many modern objects or how they are put together and their degree of attachment to other objects. In this situation it would appear that people see colors and shapes first and their interpretation of what is an object is dependent upon their prior knowledge and experience. It also seems as though perceptual relativity does apply to objects as objects can only be the sum of their properties and perceptual relativity applies to all of the properties of objects.

There are three essential points that need to be understood when trying to understand the universe we perceive. The first is that what we see varies with the sensory apparatus used to make the observation. The second is that it is not possible to get past the veil of perception to see things how they are; if there is any particular way that they are. The third is that there is no special or standard or normal means of or conditions of observation that can show us how things really are. If there is any special or single way things are, it is not available to us. One problem with many arguments about our perception of the universe is that they assume we can somehow know how things really are, when this is in fact impossible.

An example of this is provided by Don Locke (Locke, 1967, 98-101) where he suggests that the real colour of something is how a thing looks to "normal perceivers" under "standard conditions" of observation. Standard conditions of observation would for example be in daylight, with no interfering sources of light and no coloured objects between the perceiver and the object being observed. The standard conditions of observation are those that are most common. A normal perceiver would be a human being with normal vision, for example a person who was not colour blind.

There are however problems with this view. Colors still look subtly different at different times of day, they look different on cloudy days and they vary depending on whether the observer is inside 
looking out, outside looking in or in the shade and upon a host of other variables. The brightness of an object will vary under these different conditions due to the different amount of light available at any given time. While there may be an average amount of light available there does not seem to be a normal amount of light. Colors will also vary with the angle at which the light is reflected off an object and it is hardly likely there is any normal or standard angle for the reflection of light.

A second problem is that normal perceivers will still perceive colour slightly differently from each other. They might all agree the colour is red but if examined sufficiently closely it would be discovered they are still not seeing exactly the same colour. It is only the width of the concept of "red" that allows it to encompass a range of different visual stimuli all of which we describe by using the term "red". Each perceiver will perceive a slightly different version of "red" when seeing a "red" object. There may be an average perceiver but it is doubtful that any perceiver is any more normal than any other perceiver.

A third problem is that some species are normally colour blind and still others are able to distinguish a wide range of colors, some of which are not observable by human beings. This means that the same object will appear differently coloured to normal perceivers of different species, so it is hard to say it has any real colour. There seems to be no reason to claim that the human view of an object's colour should prevail over those of any other species.

The fourth problem with Don Locke's attempt to find real colors is that normal and most common does not mean, entail or imply reality. Something may be normal or most common but that does not mean that it is necessarily real or leads to reality. Locke's choice of normal and most common are simply arbitrarily selected conditions to which he chooses to claim possess some special status that makes them the conditions under which we are able to perceive real properties. However there does not seem to be anything so special about those conditions that enable us to say that properties seen under those conditions are real while properties seen under other conditions are not real. It does not follow from normal perceivers and standard conditions that you will get real colors. Normality and most common have nothing to do with reality. It seems there are no normal perceivers or standard conditions under which we are able to perceive the real properties of objects, such as their real colors.

There is a misunderstanding of perceptual relativity and idealist philosophies contained in many publications. It has been suggested the fact that a person can close his or her eyes and then reopen them and see essentially the same view shows the existence of an external reality. Things are usually assumed to continue to exist when not observed. If I cease to look at a table, I lose my sense perceptions of the table, but if I look back again I will see the table again. It is however going beyond what we can tell from such an experiment that the table exists when it is not being observed. All we can say is that the sense perceptions disappear when you cease to look at something but that they return when you look at it again. If we look at the table again, after having turned away from it, we will see it again. This is known as the permanent possibility of perception, that, whenever a certain sensory apparatus is directed at a certain point in space and time it will always produce the same sense perceptions. This is not evidence that a table exists, other than as sense perceptions, but that the relationship between the observer, the table and the conditions of observation is consistent over time. (Mill, 1889, 230-234). This argument that consistency in sense perceptions over a period of time shows an external world is fallacious. It is perfectly possible that such a person was seeing sense perceptions without there being any external reality behind them, both before the person closed his or her eyes and after reopening them. 
It has also been suggested that because a person is able to perceive the same objects with different senses, this shows the objects exist as part of the real world. This argument claims that coherence among our senses shows an external world. This is the Dr Johnson mistake, when Dr Johnson sought to refute Berkeley's arguments for idealism by kicking a large stone and one supposes getting a sore foot. Coherence among our sense perceptions does not show there is a world independent of observers as it is perfectly possible that the visual sensation of the stone and the foot feeling the stone are both sense perceptions. Adding sense perceptions together, does not equal an objective reality, it just gives you additional sense perceptions. Coherence among sense perceptions is caused by the senses all having evolved to have the same purposes of helping the organism find food, avoid predators, find mates and for navigation. A rotting piece of fruit, for example, will smell bad, taste bad and look and feel different from ripe fruit.

Other arguments concern a person's perception of other people who appear to be similar to him or her and they communicate with him or her and say they see very much the same world that he or she sees. This argument claims that a similarity in sense perceptions with other people's shows there is an external world. A similar argument to this involves the example of people driving cars at traffic lights, where the drivers all receive the same sensory perceptions of red and green lights. It is suggested that it is much more likely that the lights really exist than by coincidence the driver's brains and consciousness all change in similar ways so they all stop and go at the right time. However it is clear that all drivers are simply seeing very similar but not identical sense perceptions. Idealists would answer that a person sees things similar to what other people see, just means that he or she has obtained additional sense perceptions and that these people say they see a similar world to his or hers merely shows that conscious beings with similar sensory apparatus will see a similar view of the world. This does not mean there is a real world out there, all the people may simply be perceiving sense perceptions. The problem is most people think in absolutes. It is all or nothing. Either people see exactly the same thing or their view is completely different. This amounts to a false dichotomy, a logical error, where people are presented with the belief all humans see an identical world or their worlds are completely different and random. In actual fact people with similar sensory apparatus will obtain similar but not identical views of the universe.

It is suggested these arguments, involving consistency of sense perceptions over time, coherence among sense perceptions and similarity of sense perceptions with those of others, naturally points to an external reality. However all these arguments are just as consistent with the view of an observer created reality as they are with an independent external reality.

The assumption that the observer dependent universe is random and chaotic is wrong and this can clearly be seen from our brief study of other animal sensory apparatus and their sensory worlds. Each species world is coherent and consistent over time and the world of each individual within a species is similar but not identical.

An observer dependent universe is not a universe of ghosts, phantoms, illusions and randomness. An observer dependent universe is a physical, solid, tangible universe with coherence and consistency. Properties such as physicality, solidity and tangibility are given to us by our sensory apparatus; that is how we know about them. They are subject to perceptual relativity and can be every bit as much a part of an observer dependent universe as an observer independent universe.

One consequence of perceptual relativity and the absence of any normal conditions of observation and our inability to get pass the veil of perception is that it is necessary to distinguish between various different types of reality. Unperceivable reality, or noumena, due to our inability to get pass the veil of perception, is something we cannot know about and we cannot even be sure it 
exists at all. Unperceivable reality is also irrelevant to us except as being a contributing factor in the creation of perceived reality. It is perceived reality which is the world in which we live. Perceived reality comes in maybe an infinite number of forms and is produced by the inter-relationship of the perceiver's sensory apparatus, the conditions of observation and whatever, if anything, that lies beyond the veil of perception. Perceived reality cannot exist without an observation being made. Unperceivable reality can exist independently of observers. When one talks about an observer dependent universe, it is perceived reality which one is talking about.

\section{Reid's solution}

A different view of the problems posed by perceptual relativity has been offered by Thomas Reid (1710-1796) who suggested that the table had a different appearance when observed from different positions, and that it changed with perspective is exactly what you would expect from a real table. Reid distinguishes between the real and apparent magnitude of the table. The real magnitude of the table could be obtained by applying the rules of geometry and perspective to the apparent magnitudes derived from the sense of sight.

Reid considered that the table appears to vary in size and shape depending on the position of the observer shows only variations in its apparent magnitude, but not its real magnitude. The table's real magnitude may be a rectangle of 1 metre by 2 metres but it will appear to become smaller as I move away from it, but it's real magnitude will always remain a 1 metre by 2 metres rectangle. The relationship between real and apparent magnitudes can be determined by the rules of geometry and perspective. If the table and the observer are placed in any particular positions, it is possible to calculate the apparent magnitude of the table from any position by using the rules of geometry and perspective. When given the apparent magnitude of the table and the position of the table and the observer it is possible to calculate the real magnitude of the table.

There are some problems with Reid's theory. Reid cannot show why the mathematics based on the measurements of the shape made at the point where the shape is, which are points at which no observer can be present at the same time, as no observer can be at all four sides of the shape at the same time, should prevail over the mathematical description of the shape which takes into account the position of the observer and the angle from which the observer sees the shape. The mathematics are equivalent in both cases and there is no more reason to believe that Reid's real magnitude is any more real than the apparent magnitudes, than to believe seven is more real than three plus four. Seven and Reid's real magnitude may be simpler than three plus four and the apparent magnitudes but that does not make them more real. Simplicity and reality are quite different concepts and it is not the case that that simplicity entails or leads to reality. It is also inevitable that some descriptions of the size of the table will be simpler than others and that there will be a simplest description, but there seems no reason why the simplest description should have any special status.

A further problem is that what Reid calls the real shape is one which can never actually be observed. It exists only as an abstract mathematical idea. However, can a figure which is never seen, a figure that is only a mathematical construct, be regarded as real? Reid is trying to say there is a world external to our minds, and a mathematical construction, a rectangle that is never seen cannot possibly provide most realists with the external world they believe exists. The Reid view of nature is a God like view, he looks at nature from a single privileged position and that is from a position no human being can ever occupy. In a sense it is the view from nowhere. It is a position akin to that Newton took when describing his cosmology and can be compared to Einstein's view of the universe which describes it from positions in which human beings could actually see the universe. 
The biggest problem for Reid is that his theory rests upon the correctness of the geometry he proposes to use to discover the real table. Reid's geometry would have been classical Euclidean geometry which assumes flat three dimensional space. If this assumption is wrong then we are unable to use Euclidean geometry to discover the real table, even if all the mathematics based on the assumption, are correct. In the 19th century various Non-Euclidean geometries were proposed by mathematicians such as Carl Gauss, Nikolai Lobachevsky, Janos Bolyai and Bernhard Riemann. The Non-Euclidean geometries suggested space could be curved rather than flat. Since the publication and acceptance of general relativity, we would now consider we are living in a world of curved, four dimensional space-time. In principle, it should still be possible to use Reid's theory using the geometry of curved, four dimensional space-time, to arrive at his real table. However while the mathematics may be reliable if the calculations are correctly made, we still would not be led to the real table, if the assumptions that the mathematics rest upon are unreliable. The view that geometry and the assumptions upon which it is based, are dependent upon our sense perceptions is quite common place. Morris Kline states:

The dominant view today as to the nature of mathematical activity is, then that the concepts and axioms are derived from experience, the principles of reasoning used to deduce new conclusions were most likely derived from experience and insofar as the applicability of mathematics to the physical world is concerned, the conclusions must be checked against experience. (Kline, 1959, 472).

Albert Einstein took the same view in his lecture on Geometry and Experience when he said:

As far as the laws of mathematics refer to reality, they are not certain; as far as they are certain, they do not refer to reality. (Einstein, 1923, 25-26).

These assumptions, whether of flat three dimensional space, or curved four dimensional space-time, are necessarily based upon our observations of our world and as we have seen these observations are not necessarily reliable and may vary with the observer.

There are an infinite number of potential geometries that could describe space in the world external to the human mind. As Henri Poincare said any physical phenomena can be explained in an infinite number of ways with space possibly being of any number of dimensions and shapes. It is only our sensory apparatus that makes space appear to us to be flat and three dimensional. Due to this we do not know which geometry, if any of them, is correct, so we are not able to use the rules of geometry to discover the real table.

General relativity is the accepted view of the universe and it considers the angles, area and length of the sides of the table will vary with the type and degree of the curvature of space-time. The curvature of space-time varies with the strength of gravity at any particular point in the universe. The strength of gravity varies at different points in the universe, so the curvature of space-time varies with it and so will the shape and size of any given object. As bodies which exert gravitational force are constantly moving through the universe, the strength of gravity and the curvature of space-time at any particular point are constantly changing. It would seem that the size and shape of the table would vary at different points in the universe which suggests the table has no single size or shape.

It is not just the shape of space that is problematic. Space appears to be three dimensional but there has been considerable scientific speculation in recent years that there may be more than three dimensions of space. Superstring theories have suggested there may be ten or twenty six dimensions, these additional dimensions being known as hyperspace. The existence of the extra spatial dimensions will considerably simplify the laws of physics and all matter and forces in the universe can be 
explained as vibrations in hyperspace. Obviously we are unable to see these extra dimensions. It has been suggested the extra dimensions are curled up into a space so small we are unable to detect them. It would seem impossible for us to visualize more than three dimensions. It is however possible to image life in one or two dimensions. In a single dimension a being could only see and move along a line, while beings in two dimensional space would be able to see and move across a plane having both length and breadth. Such a two dimensional being would have no concept of height and if a three dimensional being reached into the two dimensional world and lifted a two dimensional being out, it would appear to the other two dimensional beings that their friend had disappeared into thin air. The two dimensional being carried into a three dimensional world, would only be able to see a two dimensional slice of everything in the three dimensional world. If two dimensional beings could only see in two dimensions and could not conceive of a third dimension, even when such a third dimension exists, this would suggest that a fourth or more dimensions could exist even though third dimensional beings (such as us) could not see the other dimensions or conceive of their existence.

How space and shapes are seen depends upon the nature of the sensory apparatus making the observation. Animals with binocular vision, such as humans, see in three dimensions partly due to binocular disparity, which is the difference between the ways in which each eye sees an image. Many animals with two eyes see space and shapes differently as the placement of their eyes on the side of the head means much less binocular vision than is available to humans, so their perception of depth is different from the human perception of depth. Animals such as rats do not see the clear images humans see as their visual acuity or clarity of vision is much less than human visual acuity. Visual acuity is measured by cycles per degree ( $\mathrm{cpd}$ ) which is the number of distinct lines that can be seen within a degree of the visual field. Human acuity is $30 \mathrm{cpd}$, while rat's cpd is 1 for normally pigmented rats and 0.5 for albino rats. This results in rats living in a world of blurry images, which means their perception of shape is quite different from the human perception of shape. (www.ratbehavior.org).

There is sometimes considered to be a difference between our perceptions of space and qualities such as colors. Colors are considered to be secondary qualities of objects in that they are sense dependent as they vary with the sensory apparatus used to observe them and with the conditions of observation. Qualities such as shape and size are considered by some to be primary qualities of objects in that they are not considered to be sense dependent and exist independently of any observer. The reason colors are considered to be secondary qualities is because we note that the color of an object will look different to the same observer under different conditions of light and to different observers under the same conditions of light. We also note that some people may be partially or totally colour blind and some animals can see more colors than humans and others will see less colors or may be naturally color blind. The distinction between primary and secondary qualities does not exist as Berkeley noted shapes are dependent upon colors as if colors are removed from an object, the shape of the object and the object itself will disappear. Shapes are simply caused by the placement of different colors.

Our observations of space are different from our observations of color. All humans under all conditions see space as flat and three dimensional. But some animals, for example those who can only detect the difference between light and dark, will not see three dimensions, while other animals who rely on smell, taste and sound as their principal senses, may well not be able to perceive three dimensional space. Humans perceive three dimensional space through the sense of sight and possibly touch but animals more used to other senses may not be able to perceive three dimensional space. This means that perceptions of space vary with the sensory apparatus used to perceive it. What we are able to show is that we see space as flat and three dimensional and some other animals probably cannot see in three dimensions and that science suggests space is very likely curved and possibly has more than three dimensions. This suggests our naked senses give us a false view of space if one accepts the 
scientific view or at least a doubtful view if one accepts there is evidence for both views of space. It is not clear whether there is a single correct view of space and even if there is, it is not clear what that correct view is. Without knowing how many dimensions space has and the shape of space it is not possible to know the real size and shape of things in accordance with Reid's theory. It is probable that space is entirely sense dependent and that things either have no single size and shape or that size and shape are in principle unknowable to human beings whose only access to the physical world is through a sensory apparatus of doubtful reliability.

There is something circular about Reid's argument. His theory relies on the certainty of geometry in order to pass the veil of perception and to allow us to discover the real world. However the geometry itself is based upon our observations of our perceived world and so is subject to all the problems that perception of our world involves. Our view of space itself is dependent upon our sensory apparatus and there is no reason why other beings with different sensory apparatus would not see space as being quite different from the way we see it. They might for example see curved space or possibly other dimensions. Different sensory apparatus may provide different observers with totally different concepts of space. Different concepts of space will result in quite different geometries. The different geometries when applied to our sense perceptions of the table will each produce a different real table. There is no limit to the number of geometries that may potentially exist, so there is no limit to the number of potential tables that may exist. This shows that Reid's method does not lead us to any single real table or any single reality. The very fact that three dimensional flat space has been replaced by curved four dimensional space-time shows the unreliability of the assumptions underlying our view of space. Our geometry may be analytic and true by definition, but the assumptions upon which it is based are not and are subject to all the problems of perceptual relativity and perceptual unreliability.

The problem for Reid is that size and shape are as dependent on our sensory apparatus as are all other qualities. It is perfectly conceivable that there could be beings that have no concept of size and shape simply due to not having the appropriate sensory apparatus to detect size and shape. A blind being with only the senses of smell and hearing could be such a being. This suggests that qualities such as size and shape are as equally sense dependent as qualities such as colour. Colour is a sense dependent quality because the ability to sense colour requires the presence of cones within the observer's eyes. However the sense dependence of size and shape is not based on certain qualities of the sensory apparatus as it is for colour, but on the very existence of the sense of sight. Reid's theory of perception probably seemed quite plausible until the discovery of non-Euclidean geometry in the 19th century and the development of general relativity in the early 20th century and the recognition of other species perception in more recent times.

Some would argue that size and shape could be understood through the sense of touch. This however would recreate the situation of the four blind Indians trying to understand what an elephant is like. They don't know whether what they are touching is the full elephant or only part of it. Touch gives a quite different view of space than is provided by sight because touch provides different information about space than sight provides. In particular it does not give us much information about distance. Sight extends to long distances while touch provides only short distance information. Which is the true view of space, that provided by our eyes or that provided by touch? Neither of them. If you say sight provides us with the better view because it provides us with more information it can be pointed out that the fallibility of sight is a large part of what this paper is about. To sum up, touch is a poor way of telling us about space and gives a different account from that provided by sight. However, even if the two senses give us the same view of space that would not mean they give the true view. Coherency among senses is not the same as truth or reality. Science, more specifically general relativity, tells us space is curved and neither sight nor touch says space is curved, so it appears neither sight nor touch provide good information about space. 
The conclusion it seems necessary to draw is that there is no single objective reality that we can be aware of. It may be that such a reality exists, but we can never know whether this is true or not. There seems little point in speculating about that which we cannot know. We can only know the world through the sensory apparatus available to us and it seems clear it provides us with a variety of perceptions many of which seem contradictory and may only be explained by an acceptance that there is a variety of worlds each existing as a result of a relationship between the sensory apparatus, the conditions of observation and whatever (if anything) that is out there. Different species will each live in their own sensory worlds and individuals within species will each live in similar but not identical worlds. Objects will not be part of a reality knowable to us, unless they are perceived. When observed they take whatever form the observer's sensory apparatus is able to give them, so they acquire their properties only when an observation is made. We are not able to get past the veil of perception to see what they are really like.

\section{Quantum Mechanics}

The problem of perceptual relativity does not just arise in the situations we have been looking at. It also appears to exist when making observations of the quantum world. Quantum mechanics, quantum physics and quantum theory all mean the same thing and describe the strange world of subatomic particles. The very name of some of the publications on quantum mechanics gives some idea of the extraordinary nature of quantum mechanics. The Mystery of the Quantum World, Where does the weirdness go, Particles and Paradoxes, The Ghost in the Atom, Quantum Physics: Illusion or Reality, Quantum: A Guide for the Perplexed gives some idea of the weirdness of the quantum world. This weirdness relates to certain matters which emerge from various experiments performed using quantum entities and electro-magnetic energy. There are a number of such experiments, but there are six main experiments. They are the double slit experiment, the EPR-Aspect experiment, the Stern-Gerlach experiments, polarization experiments, barrier experiments involving quantum tunnelling and a thought experiment known as Schrodinger's cat experiment.

No one really understands the quantum world. Richard Feynman, a Nobel Prize winning physicist, said "I think I can safely say, that no one today understands quantum mechanics" while Sir Roger Penrose said quantum mechanics "makes absolutely no sense" even though it agrees with all the results of the quantum mechanical experiments. Niels Bohr said "Anyone who is not shocked by quantum theory has not understood it."

The reason why people are not able to make sense of quantum mechanics is because when experiments are conducted using quantum particles and electro-magnetic radiation the particles and radiation do not seem to exist until an observation is made. Heinz Pagels in The Cosmic Code p 144 states "There is no meaning to the objective existence of an electron at some point in space... independent of actual observation. The electron seems to spring into existence as a real object only when we observe it." The act of observation brings the particle into existence. Prior to an observation the particles and radiation behave like waves, which have no physical existence, but after an observation they come into existence and behave like particles.

Needless to say, considerable efforts have been made to explain these puzzling quantum mechanical effects. The most generally accepted interpretation is known as the Copenhagen Interpretation, so called because a lot of work on its development was done in that city. The Copenhagen Interpretation has a number of aspects such as taking into account the complete experimental set up, including the observer and the measuring apparatus, the collapse of the wave function, the uncertainty principle and complementarity. 
The Copenhagen Interpretation emphasizes it is necessary to take into account the whole experimental apparatus, including the observer. It is the observation that brings the quantum entity into existence and you can alter what is observed by changing the experimental set up. One experimental set up shows the wave aspect of quantum entities, while another will show the quantum entities as particles.

The collapse of the wave function is a process which occurs whenever an observation or measurement is made of a quantum entity. It involves the quantum entity's wave function collapsing to form a particle. The wave function is not a real physical wave like waves in the ocean. Rather the wave function is an abstract mathematical concept based on probability. Before an observation is made, the quantum entity is considered not to exist and to be in a superposition of states and has no definite properties such as position or momentum.

The uncertainty principle concerns the impossibility of obtaining precise measurements of certain pairs of properties of quantum entities. The example usually given is that of the position and momentum of an electron, the principle stating the more precisely we try to measure the position of an electron, the less precisely we will be able to measure its momentum. The mathematics of quantum theory makes it clear that quantum particles simply do not have a precise position and a precise momentum. A quantum particle may have a precise position but then it will not have any knowable momentum at all, or it may have momentum, but its position will not be knowable. (Gribben, 1984, 157).

Complementarity is simply the idea that the quantum world can be seen in alternate ways. One can for example see it in terms of waves or in terms of particles. Both the wave view and the particle view are necessary to understand the quantum world. They can be seen as different sides of the same coin. Any experiment designed to show waves, will show waves, any experiment designed to show particles will show particles, however no experiment will be able to show both wave and particle pictures of the quantum world at the same time. It should be mentioned however that an experiment has been performed in Japan which shows the same photons acting as both wave and particle at the same time. (Gribben, 1995, 119-120). However such an experimental result would still be quite consistent with the view that our perception of reality is determined by a relationship between the observer, the observed and the condition of the observation. The experiment acts as an extension of our sensory apparatus and just as changes in our sensory apparatus will give us a different view of that which is observed, changes in an experiment will produce a different view of whatever is being observed. In these circumstances there is no great surprise in seeing quantum entities appear as particles and waves at the same time. Experiments may well produce apparently contradictory results much in the same way that the size and shape of objects in the macro world vary with the position of the observer and colors vary depending on the sensory apparatus used to observe them and the light conditions.

The consequences of the Copenhagen Interpretation are that the observer plays a critical role in determining how the world is. The behaviour of quantum entities depends on whether an observation is being made. If it is, then the wave function collapses and they behave as particles. If no observation is made, then quantum entities behave as waves. The waves however are probability waves which do not have any material form; they are just mathematical concepts. They do not really exist. Niels Bohr, the most prominent proponent of the Copenhagen Interpretation, considered that whether you get waves or particles, depends on the whole experimental set-up, including the quantum entities and the human observer.

There has been much controversy about what is meant by an observation causing a quantum entity to come into existence. The controversy is caused by ambiguity in the meaning of terms such as 
existence and reality. The correct interpretation is that when we say that quantum entities do not exist except when observed, we mean they do not exist as part of the human sensory world, created by the human sensory apparatus, when an observation is made. The human sensory world is the world we know and live in, and is the world given to us by our sensory apparatus. There is nothing to stop quantum entities existing in other worlds when being observed in the human sensory world and when not being observed in the human sensory world. In addition all observations made by a human observer are personal to that observer, although other human observers will perceive something very similar to that which is observed by other human observers due to having very similar sensory apparatus. The evidence we perceive in our experiments which indicates the presence of quantum entities should be treated in the same way as our perceptions of tables, trees and people in the macro world.

Such a view of quantum entity existence provides a logical explanation for the quantum experimental results which is consistent with the explanation for the workings of the human sensory apparatus provided earlier in this paper. We don't actually see quantum entities, we see macro level evidence the quantum entities exist, and this macro level evidence comes into existence in exactly the same way as everything else in the macro world. The solution to the quantum measurement problem lies not in the quantum world, but in how the human sensory apparatus works in the macro world.

The problem of quantum mechanical effects is most dramatically shown by the paradox of Schrodinger's Cat. Quantum mechanical effects are assumed to apply at macroscopic level as macroscopic objects are made up of quantum entities. The cat is placed in a chamber with a radio-active substance of which there is a $50 \%$ chance one of its atoms will decay within an hour. Should the atom decay a Geiger counter will detect this event and cause a hammer to break a flask containing a poisonous gas and so kill the cat. The common sense view is that after one hour the cat will be either alive or dead. However the Copenhagen Interpretation considers that as the Geiger counter is made up of quantum entities it is subject to the quantum mechanical rules and does not exist until an observation collapses its wave function. It is not until an observer opens the chamber that the wave function of the whole system collapses and the gas may or may not have killed the cat. Before the observer opens the chamber the Copenhagen Interpretation considered the cat to be neither alive nor dead but to be in suspended animation or a superposition of states. Some believe the cat can be considered to be both alive and dead at the same time. Obviously a cat at any one time must be either dead or alive and it certainly cannot be both dead and alive. Under the Copenhagen Interpretation the cat does not exist until an observation is made so it certainly cannot be both dead and alive, it simply doesn't exist. So what is happening in the box before it is opened and the wave function collapses? One view is that the cat itself is able to collapse the wave function so the cat is never in a superposition of states. If the cat is able to do this would an ant, a bacterium or a computer also be able to collapse the wave function? Yes, it could, but only in its own sensory world. If there was a human, sometimes called Wigner's friend, in the box instead of the cat no doubt he or she will collapse the wave function. After an hour when we open the box, Wigner's friend will either report nothing has happened or we will find the corpse of Wigner's friend. There is no case of a superposition of states here but to a human outside the box the superposition of states remains. The collapse of the wave function is personal to each individual observer. If the human opening the box is not actually being observed, if for example the building was sealed off to protect the experiment, then his or her wave function will not have collapsed except in that person's own sensory world. To the people outside the building, everything within the building will be in a superposition of states and this situation continues in an infinite regression. Clearly any conscious being must be able to collapse its own wave function, in effect to be self-actualizing and to bring itself into existence. 
The Schrodinger's cat paradox is designed to show how ridiculous quantum theory is when applied to the macro world. Yet as entities in the macro world are all made up of quantum entities it would appear that quantum theory must apply in the macro world. How can a macro entity, like a cat, exist if the quantum entities that make up the cat do not exist? Furthermore the phenomenon of superconductivity shows quantum effects operating in the macro world. It is possible to observe quantum effects on macro level instruments such as superconductor rings which may be several centimetres across and are of course made up of a vast number of atoms. This means it is not possible to say the rules of quantum mechanics apply only in the quantum world. (Davies, 1980, 128-129; Lindley, 1997, 176). It appears the same set of rules should apply to both the macro and quantum worlds and the rules that should apply are the quantum rules. So macro level measuring devices such as Geiger counters, bubble chambers, photographic plates, cats and humans should not exist until they are observed.

If one accepts the standard Copenhagen view of the quantum world, quantum entities do not exist until they are observed. If one accepts the standard common sense view of the macro world things continue to exist regardless of whether they are being observed. An attempt to accept both views would mean there must be a level where the rules of quantum theory cease to apply and the rules of classical physics begin to apply. It is however hard to pinpoint exactly where this level is and why it should apply.

There may however be a way around this problem. One way of seeing the macro world is that existence or reality can be seen as a relationship between the observer, the conditions of observation and the observed. Such a view would appear to reflect the facts of perception much better than the realist view that there is a single objective reality "out there". Perceptual relativity shows the world varies with the observer and the conditions of observation creating insoluble problems for the realist single objective reality view of the world. If an external world has a single objective form, we can never know it. Rather, all we can know is the variety of worlds created by the relationship between our sensory apparatus, the circumstances of the observation and that unknown and unknowable entity, the external world, if it exists at all. This is the view of the macro world suggested earlier in this paper.

This view of the macro world is consistent with the Copenhagen Interpretation and of the results of experiments that reveal the quantum world to us. Changing the means of observation, the experiment, results in a change in what one sees, which is very like perceptual relativity in the macro world.

The view of the atom given to us by science also seems to be subject to perceptual relativity. John Gribben in Schrodinger's Cat says:

"not only do we not know what an atom is "really" we cannot ever know what an atom is "really". We can only know what an atom is like. By probing it certain ways, we find that under those circumstances, it is "like" a billiard ball. Probe it another way and we find it is "like" a solar system. Ask a third set of questions, and the answer we get is it is "like" a positively charged nucleus surrounded by a fuzzy cloud of electrons." (Gribben, 1995, 186).

Again we see that changing the method and conditions of observation changes what we see.

The uncertainty principle is consistent with such a view of reality. The uncertainty principle states, we cannot observe properties of quantum entities such as position and momentum at the same time. In other words the methods and conditions of observation determine what we will see. If we engage in one experiment we will observe the position of the quantum entity; another experiment will 
show its momentum. But as in the macro world, there are limits to what we can observe; we are not able to get past the veil of perception.

The two qualities of wave and particle appear to be contradictory as particles are limited in space, may be at rest and rebound when they collide, while waves are spread out, inherently in motion and can merge together when they collide. It would be a contradiction to say "Light is both a wave and a particle" but there is no contradiction in the statement "Light observed by experiment $a$ appears as a particle and light observed by experiment $b$ appears as a wave." The different experiment, the different method of observation, explains why the same phenomena, appears differently under the different situations. Equally it would be a contradiction to say the table before us has a number of different shapes but it would not be contradictory to say "The table has different shapes when observed from different positions." Wave/particle duality may be just another example of perceptual relativity.

The idea that it is the act of observation that brings quantum entities into existence is quite consistent with the idea that reality (or the only reality available to us) consists of a relationship between the observer, the observed and the conditions of the observation. The reason why things in the macro world, such as tables, only exist when they are observed is that the only reality known and knowable to us exists in the form of phenomena (e.g. sense perceptions) and phenomena requires the participation of an observer for it to come into existence. If the observer is not present, or looks away or closes his or her eyes then the phenomena or sense perceptions disappear. Some might argue that even though the sense perceptions disappear, the table remains. But they cannot tell us what it is that exists apart from the sense perceptions. The only awareness we have of the table is the sense perceptions and when they disappear we are left with nothing.

The situation in the quantum world differs from that in the macro world as in the macro world evolution has provided us with a set of senses that tend to give us a coherent view of that world. We are misled by this coherence into believing our view of the world is true and real. In the quantum world we have a variety of rather disjointed ways of seeing that world, so we can get contradictory and puzzling views of that world. When quantum mechanics says that light sometimes consists of waves and at other times consists of particles it just means that one sensory apparatus or experiment will show light in one state, another will show it in a different state. We have different experiments and hence different sensory apparatus so we can see various different pictures of light.

\section{Criticisms of the observer dependent world}

Understandably there are some criticisms of the idea of the observer dependent world. One criticism is contained in Paul Davies Other Worlds. He gives the example of a Geiger counter measuring the decay of a radioactive nucleus with a pointer on the Geiger counter indicating whether or not the decay has taken place. Two successive photographs are taken of the Geiger counter pointer at the time of the experiment. The first photograph is given to scientist A and pocketed; the second photograph is given to scientist B who looks at it and according to the Copenhagen Interpretation collapses the wave function of the Geiger counter pointer. Davies suggests that when A looks at his photograph and it shows the same as the second photograph given to scientist $\mathrm{B}$, there is a case of backward causation. (Davies, 1980, 134-135). This however is not necessarily the case. If one observer makes an observation and collapses the wave function of any quantum or macro entity, he or she does not collapse it for any observer other than his or her self. In short the collapse of the wave function is a personal matter between the observer and the observed. When scientist B looks at the 
photograph it only brings the Geiger counter pointer into existence for scientist B. Scientist A remains ignorant of the result of the experiment until he or she looks at the photograph given to his or her self. The causation chain runs not from photo 2 to photo 1 but from the taking of photo 1 to scientist $\mathrm{A}$ looking at photo 1 . No backward causation is involved.

Another problem suggested for an observer dependent universe is that the observer must exist before the observer can make an observation. This means something must observe the observer to collapse his or her wave function before he or she makes an observation. Equally, who or what observes or collapses the wave function of the being that observes the original observer. This obviously goes on in an infinite regression with no solution to the problem. (Davies, 1980, 134-135; Gribben, 1984, 205-207). A related problem is when the first conscious being evolved what collapsed its wave function and brought it into being. The answer to these problems is that conscious beings are self-actualizing, they collapse their own wave function, they bring themselves into existence. The first thing any conscious being must be conscious of is itself or possibly consciousness of self occurs simultaneously with the first observation of anything external to itself. If a being can observe something external to itself, there is no reason to believe it cannot observe itself and that both observations can be simultaneous.

A further problem sometimes posed for the observer dependent world is that there was once a time when there were no human or other observers of any kind so how could events such as the big bang and the formation of stars and galaxies take place? This question was put to Rudolf Peierls in The Ghost in the Atom (Davies \& Brown, (ed), 1986, 75) who suggested the information we had now of such events allows us to be aware of what has happened before observers were present and so in a sense we are still able to observe the past. More particularly what is being talked about when an observer dependent universe is discussed is the world of phenomena, which is the only world available to us. This however does not exclude the possibility of things happening outside of our world. Our world, the phenomena and sense perceptions we perceive, is limited to the information that can be provided to us by our senses and to any additional information that may be obtained by scientific experiment. There is however good reason to believe there may be plenty going on beyond our world and things such as the big bang and the formation of galaxies before we became aware of them would be examples of such things beyond our sensory perception and scientific experimentation.

The view that reality consists of a relationship between the observer, the observed and the conditions of the observation has a number of advantages. It avoids the necessity of having different rules for the macro and micro worlds; having to draw a line between them and having to explain why the two worlds are different. It avoids the problem of how macro entities such as people exist while unobserved, while their constituent subatomic parts do not exist until they are observed. It explains the puzzle of perceptual relativity in the macro world and gives the same account for puzzles in the quantum world, such as wave/particle duality.

\section{Alternatives to the Copenhagen Interpretation}

If the Copenhagen Interpretation is considered weird, one should consider some of the alternative theories proposed to explain the behaviour of the quantum world. The "many worlds interpretation" presents, a view of the universe that involves many worlds (probably an infinite number of them) existing across time and parallel to our own world but totally cut off from it and all the other worlds. The many worlds interpretation accepts the existence of wave functions, but instead of the wave function collapsing whenever an observation is made, a different world comes into 
existence for each possible outcome of the observation. If there are two possible outcomes then two separate worlds will come into existence both virtually identical except that one will contain one outcome of the measurement process and the other the other outcome. This involves a continual proliferation of worlds, new worlds continually being created every time an event takes place with worlds branching out from previous worlds like the branches of a tree. The many worlds interpretation has its critics mostly concerning the vast and possibly infinite number of worlds that exist under this interpretation. Countless universes constantly being created due to quite trivial events, seems weird. Such endless creation of worlds positively invites the use of Occam's razor.

A further alternative to the Copenhagen Interpretation is the "transactional interpretation" of quantum mechanics developed by John Cramer in the 1980's. This interpretation involves waves travelling both forwards and backwards in time. Quantum waves traveling backwards in time are contrary to common sense and suggest that events may precede their causes. A further problem with the transactional analysis is that it appears to be contrary to notions of free will in that every photon emitted has its future settled for it.

Quantum logic arises from a belief that the paradoxes of quantum mechanics are caused by our attempt to impose classical logic on the quantum world. If we could find the right logic to use when dealing with the quantum world it may be that the paradoxes will dissolve. The supporters of quantum logic tend to compare quantum logic with non-Euclidean geometry pointing out that our empirical experience of physics has lead us to adopt non-Euclidean geometry and equally our empirical experience of quantum mechanics should lead us to abandon classical logic and adopt quantum logic. Quantum logic comes in a variety of forms, some saying it applies to both macro and quantum worlds; others suggesting it just applies to the quantum world. The use of quantum logic allows quantum mechanical realism; quantum entities exist and they have determinate qualities prior to measurement. Quantum logic would appear to eliminate any trace of conventional rationality from quantum mechanics. Quantum mechanics is meant to be a science and the use of quantum logic would destroy quantum mechanics as a science. It is hardly allowable to use a new logic just because the results of experiments seem odd. If quantum logic could be used in quantum mechanics, it is hard to tell why quantum logic or any other logic should not be used in any other area of science and if any logic can be used in any area of science, then you may get results which would destroy any chance of science giving us any understanding of the world. As Heinz Pagels suggests adopting quantum logic is like inventing a new logic to show the earth is flat, when provided with evidence that it is round. (Pagels, 1952, 167). Anything we don't like we just adopt a new logic to get rid of it.

The Copenhagen Interpretation itself has apparently weird aspects. The view that an act of observation brings the world into existence, the impossibility of measuring certain co-related properties of quantum entities such as the position and momentum of particles are all contrary to our normal experience of the world.

It appears all explanations for the quantum world involve weirdness. As David Lindley suggests you can push the weirdness round by adopting one view or another, but you cannot get rid of it. (Lindley, 1997, 121, 124). All interpretations of the quantum world are weird in one way or another. It seems quite apparent that whatever explanation one accepts for the extraordinary behaviour of quantum entities one is inevitably required to accept an explanation that seems weird.

Many scientists seem reluctant to abandon realism, usually it seems due to a misunderstanding of what the abandonment of realism would mean. They feel the abandonment of realism would trivialize science and turn the foundations of science to sand.

However this is not the case as science can be carried out exactly as it has been in the past regardless of whether one accepts realism or not. As David Lindley suggests, what is necessary for 
science is that scientists doing the same experiments will get the same results. There is no need to assume that those results relate in any way to an underlying single objective reality. The assumption of an underlying single objective reality is totally unsupported by any experimental or other evidence and is described by Lindley as a leap of faith, rather than of scientific necessity. In fact there is plenty of evidence from quantum mechanics and from perceptual relativity in the macro world to suggest there is no single, observer independent reality, knowable to us. The facts support an observer dependent world, only prejudice or "leaps of faith" support the observer independent realist world. David Lindley distinguishes between what he calls weak objectivity (the observer dependent world) where all scientists doing the same experiments will get the same results, and strong objectivity (the observer independent world) where scientific experiments all show the same underlying reality which exists independently of any observation made of it. Lindley notes that weak objectivity is essential to the functioning of science; it is the minimum standard that scientists must accept in order to do their work. Going beyond weak objectivity to strong objectivity is unnecessary, but strong objectivity is an assumption traditionally made in classical physics, but is not demanded by classical physics. The jump from weak to strong objectivity is a leap of faith, rather than of scientific necessity. (Lindley, 1997, 159-161). It seems the acceptance of the idea of an observer dependent reality may not be such a disaster for science at all.

The Copenhagen Interpretation of quantum mechanics has extraordinary similarities with the interpretation of the macro world suggested earlier in this paper. Our interpretation of the macro world suggests what is observed depends upon the sensory apparatus used to observe it, the conditions of observation and the point in space-time that is observed. This seems to be much like Bohr's statements that when observing the quantum world you had to take into account the whole experimental set up, including the observer. Altering the experimental set up is the same as altering the sensory apparatus and conditions of observation in the macro world.

The view that things don't exist until an observation is made seems very much the same as the Copenhagen view that quantum entities such as photons and electrons only come into existence when an observation is made. Equally properties such as colors only come into existence in the macro world when an observation is made and properties such as photon polarization and electron spin only come into existence in the quantum world when an observation is made. If, as is clear from earlier in this paper, tables, trees, people and everything else in the human sensory macro world only come into existence when observed, then our observations of the effects in our experiments that tell us of the presence of quantum entities must also only come into existence when an observation is made. How could it be otherwise? as our observations of the results of our experiments are macro level observations seen in our macro level experimental apparatus and come to us via our sensory apparatus. It is not in the quantum world, or the experimental apparatus, that the explanation for quantum entities only existing when observed can be seen. It is the way the human sensory apparatus creates the human sensory world that causes quantum entities, as shown by the effects in macro level experimental apparatus, to come into existence and acquire their properties. This may possibly solve the quantum measurement problem.

Perceptual relativity clearly applies in the macro world but wave/particle duality suggests it also applies in the quantum world and complementarity is arguably just another name for perceptual relativity. It is impossible to get past the veil of perception in the macro world to see how things "really" are. The uncertainty principle in the quantum world looks very much like an example of how we cannot get past the veil of perception. 
In quantum theory we are often considered as only obtaining a snapshot by snapshot or observation by observation view of the quantum world. This is a view without any continuity which is considered to be different from the macro world where we have continuity. In the macro world we see by reflected light, by photon after photon which gives an appearance of continuity, but is not really continuous. We get a view similar to watching TV which appears continuous but we know can be slowed down to a frame by frame view where we can see there is no continuity. The appearance of continuity in the macroworld is caused by the workings of our sensory apparatus which gives apparent continuity, but in reality photons reach our sensory apparatus one by one so there is a discontinuity in the macro world as well as the quantum world, even though in the macro world the discontinuity is hidden from us. The external points of reference Bohr considered we have in the macro world such as space, time and causality are just creations of our sensory apparatus and a different sensory apparatus would not have available those points of reference.

The Copenhagen Interpretation has long been the orthodox view of the quantum world and this is not surprising considering how weird the alternatives are. However realism has usually been assumed in the macro world, but given the modern research into animal senses, neurology and cognitive psychology, realism must inevitably cease to be a serious explanation of the macro world. It seems quite obvious the macro world is sense dependent and the orthodox interpretation of the quantum world postulates a sense dependent world as well. This suggests the same rules can apply to both the macro and quantum worlds, which eliminates the need for a dividing line between the two worlds.

The first two paragraphs of the quantum mechanics part of this paper suggested the quantum world was weird and not really understandable. This is because we tend to compare it with the macro world and it seemed very different from the macro world. But we have misunderstood the macro world and when we come to understand the macro world as an observer dependent world, the quantum world does not appear weird at all. 


\section{Part II}

\section{The consequences of perceptual relativity and the observer dependent universe}

It seems obvious that we can only know the world through our senses. This could provide a limitation and a starting point for a logical process that could lead to a theory of existence or reality. We know our senses are subject to perceptual relativity, they disclose only a part of the world outside of us and they control the way the world appears to us. Due to the limitations of our senses, there are a number of worlds or realities that can be considered to exist.

A list of such realities could be:

1) noumena-unknowable. This first type of reality, if it exists, concerns whatever causes light to be reflected or vibrations in the air, the "thing in itself" or "noumena" referred to by Kant. We have no idea what this type of reality is like and in principle this world is unknowable to us.

2) the unknown world outside our sensory thresholds, potentially knowable, but not currently known. This is the reality we can obtain by extending our senses by scientific means i.e. microscopes, telescopes, logical deduction from observing the results of experiments, but have not yet done so.

3) the reality we know exists due to scientific endeavor, such as far away galaxies, bacteria and viruses and subatomic particles. This world may not currently be observed but is known from previous observations and because our world has consistency and stability.

4) perceivable but currently unperceived reality- the permanent possibility of perception in the macro world. In the quantum world this is the superposition of states described by the wave function before an observation or after an observer has ceased to look.

5) current sense perceptions in the macro world. This is the world, human beings live in and are immediately aware of. In the quantum world, this is the situation after an observation has taken place and the wave function has collapsed.

6) belief in things that don't exist-such as dog headed men, a widespread belief in medieval Europe and the vast array of gods many humans believe in.

This list is not necessarily exhaustive. All of the above, except 1),2) and 6) are subject to perceptual relativity and can be regarded as phenomena and are based upon our sensory perceptions. Our sense perceptions are all we can know, and phenomena is the only reality available to us. Phenomena has no fixed form, it changes with the sense organs used to observe it. There is no reason to believe there is any relationship at all between what we perceive in phenomena and what possibly causes the sense perceptions in noumena. If we remove the observer then phenomena will vanish while noumena (whatever that is) may continue to exist. Phenomena, is however fixed, in that if we perceive it with the same sensory apparatus and in the same conditions of observation, it will always be the same. If the sensory apparatus and conditions of observation remains the same, phenomena remains the same. This gives coherence and consistency to our sense perceptions. 
The situation appears to be that if human senses are changed in any way the human view of the world is altered. If a sense is eliminated, for example, through blindness, deafness or some other cause the person's view of the world will be different. If an additional sense is added the person's worldview is changed by the information received through the additional sense. Equally if an existing sense is improved for example sight or smell we would be able to see things we could not previously see and perceive smells that we could not previously smell. This means the world is sense dependent. If you alter the sense, you alter the world. The reality we perceive seems to be totally sense dependent and is in fact not "that which is out there" but is a relationship between "that which is out there" and our sensory apparatus and the conditions of observation. If there are an infinite number of types of senses, then there must be an infinite number of types of worlds. There seems to be no reason why we should regard the range of senses as being limited to the five human senses. That a range of sense organs much wider and different from our own exist can be seen most easily from the animal world. It is well known that many animals can sense things that humans cannot. Dogs for example can hear sounds and perceive smells that humans would be completely unaware of. This however merely involves an extension of senses that humans have. Some animals have senses quite different from anything humans possess and live in a world way beyond our imagination. Dolphins and bats have a form of sonar and radar which enables them to detect obstacles and prey. Many fish are able to generate electric currents and have organs capable of detecting any distortion in the electric field caused by objects in the electric field. Other animals such as birds, lobsters and even bacteria appear to be able to detect the earth's magnetic field and in the case of birds and lobsters are able to use this ability to assist their migrations over considerable distances.

An important property of all senses is that they have thresholds. Furthermore different senses have different thresholds, for example cats, dogs and humans will all have different visual, aural and nasal thresholds; each will perceive the world differently from the others, each will perceive things the other will not and will fail to perceive things the others will perceive. The effect of thresholds is that for each individual and each species there is a reality that each individual and species can perceive and other realities lying beyond the individual or species sense perception.

Each sensory world can be described in a number of ways. A world can be described as a set, the sum total of all sensory perceptions produced by a given sensory apparatus, will constitute the set. Within a given species there will be a great overlap between each individual's set of sensory perceptions due to individuals having very similar sensory apparatus. Insofar as individual's sensory worlds within a species vary, the variation is mainly but not exclusively caused by those individual's different positions in space and time and minor variations in their sensory apparatus. Between species there will be much less overlap due to the rather more varied sensory apparatus between species or there may be no overlap if the sensory apparatus are sufficiently different. Let us for example look at being A, a human being. Being A can see certain shapes and colors, can perceive a rather limited range of smells, can hear certain sounds, taste various tastes and feel certain things and not others. Being $\mathrm{B}$, for example a cat, will perceive some things that the human will not perceive, but will fail to perceive some things the human will perceive. The cat will certainly smell things the human cannot and taste and feel things that humans cannot and fail to taste and feel things that humans can. The end result is that there is an overlap between the humans and cats sense perceptions, but there are also some sense perceptions only the human will receive and other sense perceptions only the cat will receive. Another being might have sensory apparatus sufficiently different from those either of a human and a cat so there is no overlap between its sensory perceptions and those of a human or a cat. This can be represented diagrammatically as follows: 


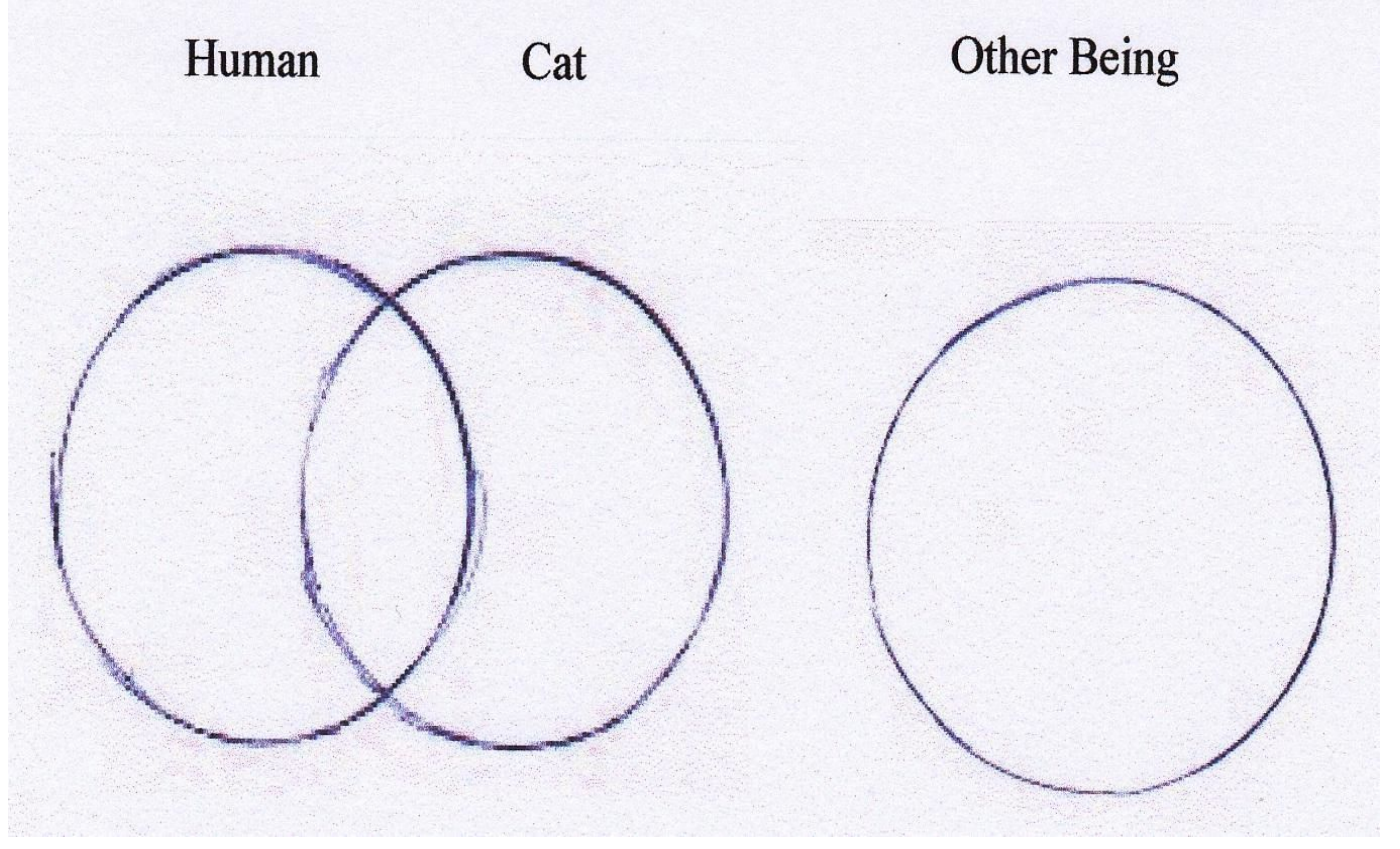

and analysed in accordance with set theory.

Sense perceptions can also be analysed mathematically by using the threshold levels for sight, hearing, smell, touch and taste all of which can be, or can potentially be given numerical values. Everything within the thresholds will be in an individual's world or species world; everything outside the thresholds will, subject to the use of science and scientific apparatus to extend sensory perceptions, be outside the individuals or species world. These threshold based worlds are similar to the set of sensory perception worlds referred to earlier and illustrated by the above diagram, but the threshold based worlds are based on the capacity of the individual or species sensory apparatus while the sets of sensory perception are based upon what the individuals or species actually perceive.

It is possible to conceive of a continuum of possible sensory apparatus, infinite, if the range of sensory apparatus is unlimited, and finite if the range of sensory apparatus is limited. In principle it should be possible to map the total possible sensory apparatus potentially available to any being and to be able to place any individual being or species (or sensory apparatus) on a particular position on that map. A separate map could be produced for each separate sensory apparatus and it may be possible to combine such maps, in some multi-dimensional way, to produce a map of the total sensory universe and each individual's and species position on the map. This would mean each being or species could be placed on a map grid showing where their sensory world is and the grid as a whole would show the total possible sensory universe.

In physics an event is something that takes place at a particular point in space and a particular point in time. It can be specified by three spatial coordinates and a single time coordinate to give its position in space-time. However for an event to have its position more completely stated it should have its position in the sensory perception continuum specified. This is because an event may occur at a particular point in space and time but may be perceivable by certain beings and not by others. More particularly an event may occur in space-time and be perceivable by human beings and another event may occur in space-time and may not be perceivable by human beings, but each event would be as real as the other. For the location of an event to be specified exactly it must not just have its position in space-time established but also its position in the sensory continuum. This would enable us to know in which or whose sensory world the event took place and what sensory apparatus may be used to detect the event. 
It is necessary to add the sensory position (or situation) of an event to its position in space-time. This enables us to explain why one observer can look at a table and see a solid brown object while another, say a scientist, would conclude that it is largely empty space sparsely inhabited by particles such as protons, neutrons and electrons. By adding the sensory position of any event, to its position in space-time, it is possible to explain why something, such as a brown table, is seen by human beings and something else such as electrons, protons and neutrons are not seen even though they occupy the same position in space-time. The sensory position of an event is the same as its position in space-time in that if one observed a point in space at different times or made observations at the same time but directed to different points in space you would observe different events. Equally with different sensory apparatus observing a single point in space-time, the observer will see a different event. One obtains a different event by changing any of the coordinates of space, or of time, or of the sensory situation of the event.

An object can be of one colour and shape in one sensory world and a different colour and shape in another sensory world. Contradictory properties become possible in different sensory worlds. To fully understand something within a sensory world, it is necessary to perceive it in context as belonging to a particular sensory world.

To understand something as well as it can be known, it must be seen in all sensory worlds. Perceiving it in one sensory world is to perceive only one view of the object, while other equally valid views are unknown. It may be argued humans are or should only be interested in their own view of the world, but a large part of the scientific endeavour has been to uncover other worlds such as the world outside human sensory thresholds. Events that happen outside our sensory world can have a great impact in our world such as the behaviour of bacteria and viruses. The Black Death involved certain events occurring outside our sensory world, the movement of certain bacteria across Asia and Europe, which had enormous consequences within our world. Knowledge of the process of nuclear fission, which occurs outside human sensory thresholds, has also had a great effect on our world, in the form of nuclear power and bombs. The ability to make things happen in the worlds beyond our sensory thresholds for example with nano-technology and to control the behaviour of other conscious beings may be important technological developments in the future. The development of robot senses may have important uses such as in surgery and in driverless cars. A better understanding of our sensory world and other sensory worlds can lead to better decisions being made ranging from better moral and ethical judgements to producing better technology. We no longer burn witches if our cow gets sick and dies, because we now believe its death was caused by viruses or bacteria. To make our crops grow, we apply fertiliser, rather than sacrifice thousands of people to our gods because we now believe crops need appropriate nutrients. A lot of unnecessary human suffering has been removed from human societies by our ever increasing knowledge of the world around us.

A consequence of perceptual relativity and the observer dependent universe is that human knowledge and awareness of the universe should be treated with considerable caution. All views of the universe, and what happens in it, should be held with a degree of scepticism. The basis for knowledge is the realisation that we know very little. As individuals we each know only a tiny bit of our total species knowledge and our species as a whole knows very little of how the universe really is. Our sensory apparatus is designed by evolution to help us in our everyday lives, but it is not designed to help us understand the universe. Yet many people hold beliefs with a degree of certainty, which is not justified, due to their ignorance of the world around them. None of us know any absolute truths, the best we can do is have rational beliefs based upon the currently available information. The fact that no human knows any absolute truths should lead to people accepting that uncertainty is a rational response to human ignorance or lack of knowledge. But most people dislike uncertainty. Certainty is something necessary for us in our everyday lives. If we did not have confidence in the world around us we would not do anything. We would be paralysed with inaction. But it is wrong to carry over this 
confidence that we need to function in our everyday life into our intellectual lives. This is a category mistake, taking something appropriate in one area of our lives, to another area where it is quite inappropriate. It goes totally against people's natural inclination, which is to believe they know everything or nearly everything. They like to feel confident; certainty, while irrational, is emotionally comforting to people. Given that truth is singular and falsehood is infinite, there is far more chance of being wrong, than of being right. This is supported by the wide range of human belief systems, whether they are religious i.e. have a supernatural element or are of a political nature e.g. democracy, communism or fascism. They can't all be right as they often contradict each other, but they can all be wrong and most likely are. The same situation exists as we have seen with the many interpretations of the quantum world. In other areas of science there is some consensus as to the true theory such as general relativity as an explanation of the universe and the germ theory of disease. But anyone who has studied the history of science knows theories which are completely accepted by one generation of scientists, will often be discarded by the next generation as new facts lead to new and better theories. But no matter how good a theory is, there is no reason to believe it true in any permanent sense. This should rationally lead to people believing in uncertainty and such a belief is likely to lead to greater acceptance and tolerance within our society.

Reality appears to consist of a vast number, possibly an infinite number, of sensory worlds. Each person has their own individual world. This is the world they perceive which is always different from the world perceived by others. Variations exist from person to person due to each person occupying different points in time and space and due to the quality of the individual person's sense organs. Each species has its own world due to the tendency for members of each species to have the same sense organs which will tend to function in a similar way within each member of the species. There seems to be no good reason for favouring any one of these sensory worlds over any other of them. It seems impossible to claim that the human view of the world has any special claim to validity when an alteration of our senses will give us different sense perceptions. How can you say what you perceive is, when the same thing can be perceived with different sense organs and it can be something quite different? If the human view was to be preferred it would be no more than a case of a human centric view of the world that is not capable of any real justification. 


\section{Bibliography}

\section{Philosophy and Perception}

Armstrong, D. M. (1961) Perception and the Physical World, Routledge \& Keegan Paul: New York Audi (ed) (1995) The Cambridge Dictionary of Philosophy, Cambridge University Press, Cambridge Ayer, A J (1956) The Problem of Knowledge, Penguin Books: London Berkeley, G (1710) Treatise concerning the Principle of Human Knowledge Broad, C D (1965) Some Elementary Reflexions on Sense Perception in Perceiving, Sensing and Knowing (ed) Swartz, R: University of California Press, Berkeley

Broad, C D (1965) The Theory of Sensa in Perceiving, Sensing and Knowing (ed) Swartz, R: University of California Press, Berkeley

Burton, Robert (1973) Animal Senses, A H \& A W Reed, Wellington, New Zealand

Chabris, Christopher and Simons, Daniel (2010) The Invisible Gorilla and other ways our intuition deceives us, Harper Collins Publishers: London

Chisholm, R M (1965) The Theory of Appearing in Perceiving, Sensing and Knowing (ed) Swartz, R: University of California Press, Berkeley

Cresswell, M J Jackson on perception in Theoria Vol.XLVI (1980) Part 2-3

Davies, P C W (1977) Space and Time in the modern universe, Cambridge University Press: Cambridge

Dowling, John E (1998) Creating Mind: How the Brain Works, W W Norton \& Co, New York Downer, John (1988) Supersense: Perception in the Animal World, BBC Books, London Einstein, A (1923) Geometry and Experience in Sidelights on Relativity, E P Dutton, New York Greenfield, Susan A (1997) The Human Brain: A Guided Tour, Basic Books, New York

Hughes, Howard C (1999) Sensory Exotica: A World Beyond Human Experience, MIT Press, Cambridge, Massachusetts

Jackson, Frank (1977) Perception, Cambridge University Press: Cambridge

Kaku, M (1994) Hyperspace, Oxford University Press: Oxford

Kelly, David (1986) The Evidence of the Senses, Louisiana State University Press: Baton Rouge Kline, M (1959) Mathematics and the Physical World, Thomas Y Crowell Company: New York Kline, M (1980) Mathematics, Oxford University Press: New York

Locke, D (1967) Perception and our Knowledge of the External World, George Allen \& Unwin Ltd: London

McKeachie, Wilbert J and Doyle, Charlotte L, (1966) Psychology, Addison-Wesley Publishing Co Inc: Reading, Mass

Mill, J. S. (1889) An examination of Sir William Hamilton's Philosophy: London

Russell, B (1912) The Problems of Philosophy, Oxford University Press, Oxford

Schiffman, H. R. (1982) Sensation and Perception, John Wiley \& Sons: New York

Smart, J. J. C. (1963) Philosophy and Scientific Realism, Routledge \& Keegan Paul: New York Winston, Robert (2003) The Human Mind, Bantam Books London 


\section{Websites}

www.ratbehavior.org

https://en.wikipedia.org/wiki/Telescope

https://science.nasa.gov/science-news/science-at-nasa/1999/features/ast20apr99 1 


\section{Quantum Theory}

Al-Khalili, Jim (2003) Quantum: A Guide for the Perplexed, Weidenfeld \& Nicolson: London

Baierlein, R (1992) Newton to Einstein, Cambridge University Press: Cambridge

Cushing, James T. \& McMullin, E. (1989) Philosophical Consequences of Quantum Theory, University of Notre Dame Press: Notre Dame, Indiana

Davies, Paul (1980) Other Worlds Penguin Group: London

Davies, P. C. W. \& Brown J. R. ed (1986) The Ghost in the Atom, Cambridge University Press: Cambridge

d' Espagnat, Bernard The Quantum Theory and Reality, in Scientific American, November 1979, $128-140$

Feynman, R, Leighton, R \& Sands, M (1965) The Feynman Lectures on Physics, Mass: Addison-Wesley

Forrest, Peter (1988) Quantum Metaphysics, Basil Blackwell: Oxford

Gibbins, Peter (1987) Particles and Paradoxes, Cambridge University Press: Cambridge

Greenstein, G \& Zajonc, A (1997) The Quantum Challenge Jones \& Bartlett Publishers: Sudbury Massachusetts

Gribbin, John (1984) In Search of Schrodinger's Cat, Black Swan: London

Gribbin, John (1995) Schrodinger's Kittens, Phoenix: London

Healey Richard (1989) The Philosophy of Quantum Mechanics Cambridge University Press: Cambridge

Herbert, Nick (1985) Quantum Reality, Anchor Press/Double Day: Garden City, New York

Hey, Tony \& Walters, P (1987) The Quantum Universe, Cambridge University Press:Cambridge

Horner, J (1987) The Description of Nature, Clarendon Press: Oxford

Hooker, C A (1972) The Nature of Quantum Mechanical Reality: Einstein v Bohr in Paradigms and Paradoxes (ed) Colodny, R University of Pittsburgh Press

Krips, Henry (1987) The Metaphysics of Quantum Theory, Clarendon Press: Oxford Lindley, David (1996) Where does the weirdness go, Vintage: London

Mermin, N. David (1990) Boojums all the way through, Cambridge University Press: Cambridge

Mermin, N David Is the moon there when nobody looks? Reality and the quantum theory in Physics Today April 1985 38-47

Rae, A (1986) Quantum Physics: illusion or reality, Cambridge University Press Cambridge

Ridley, B. K. (1976) Time, Space and Things, Cambridge University Press: Cambridge

Squires Euan J. (1986) The Mystery of the Quantum World, A Hilger: Bristol

Wolf, Fred Alan (1981) Taking the Quantum Leap, Harper \& Row: San Francisco

Zohar, Danah (1991) The Quantum Self, Flamingo: London 